Geometry $\&$ Topology

Volume 8 (2004) 743-777

Published: 18 May 2004

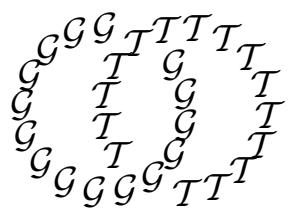

\title{
Constructing symplectic forms on 4-manifolds which vanish on circles
}

\author{
DAVID T GAY \\ ROBION KIRBY \\ CIRGET, Université du Québec à Montréal, Case Postale 8888 \\ Succursale centre-ville, Montréal (QC) H3C 3P8, Canada \\ and \\ Department of Mathematics, University of California \\ Berkeley, CA 94720, USA \\ Email: gay@math.uqam.ca and kirby@math.berkeley.edu
}

\begin{abstract}
Given a smooth, closed, oriented 4 -manifold $X$ and $\alpha \in H_{2}(X, \mathbb{Z})$ such that $\alpha \cdot \alpha>0$, a closed $2-$ form $\omega$ is constructed, Poincaré dual to $\alpha$, which is symplectic on the complement of a finite set of unknotted circles $Z$. The number of circles, counted with sign, is given by $d=\left(c_{1}(s)^{2}-3 \sigma(X)-2 \chi(X)\right) / 4$, where $s$ is a certain $\operatorname{spin}^{\mathbb{C}}$ structure naturally associated to $\omega$.
\end{abstract}

\section{AMS Classification numbers Primary: 57R17}

Secondary: 57M50, 32Q60

Keywords: Symplectic, 4-manifold, spin $^{\mathbb{C}}$, almost complex, harmonic

Proposed: Yasha Eliashberg

Seconded: Leonid Polterovich, Simon Donaldson
Received: 17 January 2004

Revised: 6 May 2004 


\section{Introduction}

Let $X^{4}$ be a connected, closed, smooth, oriented $4-$ manifold with $b_{2}^{+}>0$. For a Riemannian metric $g$ on $X$, let $\Lambda_{+}^{2}$ be the 3 -plane bundle of self-dual 2 -forms on $X$. Harmonic 2-forms are closed sections of $\Lambda_{+}^{2}$; it is known [12 that there exist metrics on $X$ for which there are harmonic 2 -forms which are transverse to the 0 -section of $\Lambda_{+}^{2}$, so that the 0 -locus is 1 -dimensional and the 2 -forms are symplectic in the complement of some circles. Here, we explicitly construct such 2 -forms and metrics.

Let us say that a connected subset $C$ in a 4 -manifold "uses up all the 3 handles" if the complement of a regular neighborhood of $C$ has a handlebody decomposition with only $0-, 1-$ and 2 -handles.

Given $\alpha \in H_{2}(X ; \mathbb{Z})$ with $\alpha \cdot \alpha>0$, let $\Sigma$ be a smoothly imbedded surface in $X$ which uses up all the 3 -handles and represents $\alpha$. In Section 3 we show that such surfaces exist, and that one can arbitrarily increase the genus of $\Sigma$ by adding homologically trivial tori while still using up all the 3 -handles. Let $c \in H^{2}(X ; \mathbb{Z})$ be a cohomology class satisfying $c \cdot \Sigma=2-2(\operatorname{genus}(\Sigma)+1)+\Sigma \cdot \Sigma$; such $c$ exist because genus $(\Sigma)$ can be increased if necessary. Then choose a $\operatorname{spin}^{\mathbb{C}}$ structure $s$ with $c_{1}(s)=c$.

Theorem 1 Given $\Sigma$ and $s$ as above, there exist a closed 2 -form $\omega$ on $X$, a finite set of signed $( \pm 1$ ) circles $Z \subset X-\Sigma$ (we will show how this sign is natural) bounding disjointly imbedded disks, an $\omega$-compatible almost complex structure $J$ on $X-Z$, and a Riemannian metric $g$ on $X$, which satisfy:

(1) $[\omega] \in H^{2}(X ; \mathbb{R})$ is Poincaré dual to $[\Sigma]$ (and hence integral).

(2) $\omega \wedge \omega>0$ on $X-Z$.

(3) $\omega$ vanishes identically on $Z$.

(4) There exists a $J$-holomorphic curve $\Sigma^{\prime}$ which is the connected sum of $\Sigma$ with a standard torus in a neighborhood of a point in $\Sigma$.

(5) There is at least one circle with sign -1 .

(6) The sign $( \pm 1)$ associated to each circle $Z_{i} \subset Z$ is the obstruction $o\left(Z_{i}\right) \in$ $\pi_{3}\left(S^{2}\right)=\mathbb{Z}$ to extending $J$ across a 4 -ball neighborhood of a certain disk $D_{i}$ bounded by $Z_{i}$ and the total number of circles in $Z$, counted with sign, is $d=\left(\left(c_{1}(s)\right)^{2}-3 \sigma(X)-2 \chi(X)\right) / 4$.

(7) The spin ${ }^{\mathbb{C}}$ structure determined by $J \mid(X-D)$, where $D$ is the union of the disks $D_{i}$, is $s$. 
(8) $\omega$ is $g$-self-dual (and thus harmonic) and transverse to the zero section of $\Lambda_{+}^{2}$ (with zero locus $Z$ ).

Remark 2 The obstruction invariant $o\left(Z_{i}\right)$ depends only on the relative homology class of the disk $D_{i}$ in $H_{2}(X, Z ; \mathbb{Z})$, not on the specific disk. We will show (proposition 26) that $o\left(Z_{i}\right)$ can also be computed by counting anticomplex (or complex, depending on orientation) points on $D_{i}$.

Note that a $\operatorname{spin}^{\mathbb{C}}$ structure $s$ is an almost complex structure on the 2-skeleton of $X$ which extends over the 3 -skeleton of $X$ ( see page 48 in 10]). Then $J$ restricted to the complement of the disks $D_{i}$ is a $\operatorname{spin}^{\mathbb{C}}$ structure $s$, and conversely, $J$ may be thought of as an extension of $s$ across the 2 -disks transverse to the $D_{i}$.

Taubes [17 has initiated a program to study the behavior of $J$-holomorphic curves in 4-manifolds equipped with symplectic forms which vanish along circles, in the hope that this will reveal smooth invariants of nonsymplectic 4manifolds. We in turn hope that our construction will produce a rich class of examples in which to pursue this program. In [16, some explicit constructions in terms of handlebodies are discussed, but not with this much generality and, in particular, not on closed manifolds. A canonical example that is in some sense diametrically opposed to our construction is the case of $S^{1} \times Y^{3}$ for a 3-manifold $Y$ : One chooses an $S^{1}$-valued Morse function $f$ on $Y$ with only critical points of index 1 and 2 , and defines $\omega$ to be $d t \wedge d f+\star_{3} d f$, where $\star_{3}$ is the Hodge star operator on $Y$ and $t$ is the $S^{1}$ coordinate on $S^{1} \times Y$. The zero circles are then $S^{1} \times p$ for critical points $p \in Y$. Here the zero circles are all homologically nontrivial; in our construction the zero circles all bound disks.

Remark 3 Honda [13] has shown that, given a metric $g$, when a harmonic 2 -form $\omega$ is transverse to the zero section of $\Lambda_{+}^{2}$, the behavior of $\omega$ on a neighborhood $S^{1} \times B^{3}$ near a component of the zero locus $Z$ is given by one of two local models. The "orientable" model is $\omega=d t \wedge d h+\star_{3} d h$, as in the previous paragraph, where $h: B^{3} \rightarrow \mathbb{R}$ is a standard Morse function with a single critical point of index 1 (or 2 ) at 0 . Thus there is a natural splitting of the normal bundle to $Z$ into a 1 -dimensional and a 2 -dimensional bundle. The "nonorientable" model is a quotient of the orientable model by a $Z_{2}$ action so that the 1-dimensional bundle becomes a Moebius strip. A positive feature of our construction is that the nonorientable model never arises.

Remark 4 In our construction the adjunction inequality is always violated in the sense that we have a $J$-holomorphic curve $\Sigma^{\prime}$ which does not minimize 
genus. When $c_{1}(s)^{2}-2 \chi(X)-3 \sigma(X)=0$, we can actually cancel the circles in $Z$ at the level of the almost complex structure, to get an almost complex structure on all of $X$ with respect to which a surface which does not minimize genus is $J$-holomorphic. This supplements examples of Mikhalkin [15] and Bohr [1]. Furthermore, this almost complex structure is compatible with a symplectic form outside a ball.

If $X$ does in fact support a symplectic structure, at first glance it appears that our construction has no hope of recovering that fact, since we always produce singular circles and we always violate the adjunction inequality. However, there is a different type of "cancellation of singular circles" that might appear. In the $S^{1} \times Y^{3}$ model described above, a flow line connecting an index-1 critical point which cancels an index -2 critical point becomes a symplectic cylinder connecting two singular circles which can be cancelled symplectically. In our construction, we could search for symplectic cylinders with this local model connecting two of our circles. To be able to cancel all the circles in this way, at least one of these symplectic cylinders would have to intersect $\Sigma^{\prime}$, so that after the cancellation $\Sigma^{\prime}$ is no longer $J$-holomorphic. Furthermore, the change would have to change $c_{1}(s)$, so that $c_{1}(s) \cdot[\Sigma]$ no longer predicts a minimal genus in $[\Sigma]$ of genus $(\Sigma)+1$. An interesting project is to search for explicit examples of this kind of cancellation.

Example 5 Consider $X=\#^{3} \mathbb{C} P^{2}$ (which cannot support a symplectic structure), with standard generators $\alpha_{1}, \alpha_{2}, \alpha_{3} \in H_{2}(X ; \mathbb{Z})$ such that $\alpha_{i} \cdot \alpha_{j}=\delta_{i j}$. Let $s$ be the $\operatorname{spin}^{\mathbb{C}}$ structure for which $c_{1}(s) \cdot \alpha_{1}=1, c_{1}(s) \cdot \alpha_{2}=3$ and $c_{1}(s) \cdot \alpha_{3}=3$ ( $s$ is unique because there is no 2 -torsion here). Let $\Sigma$ be the standard $\mathbb{C} P^{1}$ representing $\alpha_{1}$ and check that $c_{1}(s) \cdot \alpha_{1}=2-2(\operatorname{genus}(\Sigma)+1)+\alpha_{1} \cdot a_{1}$.

In our construction we first build $E$, a neighborhood of $\Sigma$, as a neighborhood $E^{\prime}$ of a $J$-holomorphic torus $\Sigma^{\prime}$ together with two extra 2 -handles. This will have a negative overtwisted contact structure on its $S^{3}$ boundary. Then we build $N=X-\operatorname{int}(E)$ with the standard two 2-handles each with framing +1 ; to attach these two 2 -handles to the 0 -handle along Legendrian knots, we need the boundary of the 0 -handle to be convex and overtwisted. One circle in $Z$ is introduced precisely to change the standard tight contact structure on the boundary of the standard symplectic 0 -handle by a Lutz twist along a transverse unknot with self-linking number -1 to achieve overtwistedness; the second circle is introduced to cancel the first circle at the level of almost complex structures, since $d=(19-9-10) / 4=0$ for this choice of $s$. The second circle corresponds to a Lutz twist along a transverse unknot with self-linking number +1 ; the self-linking numbers are exactly the signs of the circles. If we put the 
two circles in a single 4-ball, the obstruction to extending $J$ across this ball is zero.

Precisely because the total obstruction to extending $J$ is $d$ ( 0 in this case), the contact structures on $S^{3}$ coming from $N$ and from $E$ are homotopic. They are also overtwisted and therefore isotopic so we can glue $E$ to $N$ symplectically. This finishes Example 5 .

We can generalize Theorem 1 to make configurations of embedded surfaces $J$-holomorphic. Let $\Sigma_{1}, \ldots, \Sigma_{k}$ be smoothly imbedded surfaces in $X$ with pairwise intersections transverse and positive (self-intersections not necessarily positive) such that, for each $i, \Sigma_{i} \cdot \Sigma_{1}+\ldots+\Sigma_{i} \cdot \Sigma_{k}>0$. Let $Q$ be the intersection form for a neighborhood of $\Sigma_{1} \cup \ldots \cup \Sigma_{k}$ and assume that $\operatorname{det}(Q) \neq 0$. Suppose that $\Sigma_{1} \cup \ldots \cup \Sigma_{k}$ uses up all the 3 -handles. (Again, at the cost of increasing genus we can use all the 3 -handles.) Let $s$ be a $\operatorname{spin}^{\mathbb{C}}$ structure on $X$ such that:

(1) $c_{1}(s) \cdot \Sigma_{1}=2-2\left(\operatorname{genus}\left(\Sigma_{1}\right)+1\right)+\Sigma_{1} \cdot \Sigma_{1}$ and

(2) for each $i>1, c_{1}(s) \cdot \Sigma_{i}=2-2 \operatorname{genus}\left(\Sigma_{i}\right)+\Sigma_{i} \cdot \Sigma_{i}$.

Addendum 6 Given $\Sigma_{1}, \ldots, \Sigma_{k}$ and $s$ as above, there exist $\omega, Z, J$ and $g$ as in Theorem 1 with the following adjustments to the properties listed in Theorem [t:

(1) $[\omega]$ is Poincaré dual to $\left[\Sigma_{1}\right]+\ldots+\left[\Sigma_{k}\right]$.

(4) There exists a $J$-holomorphic curve $\Sigma_{1}^{\prime}$ which is the connected sum of $\Sigma_{1}$ with a standard torus in a neighborhood of a point in $\Sigma_{1}$.

(4') $\Sigma_{2}, \ldots, \Sigma_{k}$ are all $J$-holomorphic.

Example 7 Let $Y$ be a closed, oriented 3 -manifold with $b_{2}(Y)>0$ and let $X=S^{1} \times Y$. (If $Y$ does not fiber over $S^{1}$ it is not known, in general, whether $X$ supports a symplectic structure or not.) Let $\Sigma_{1}$ be a homologically nontrivial surface in $Y$ and let $\Sigma_{2}=S^{1} \times \gamma$, where $\gamma$ is a knot in $Y$ transversely intersecting $\Sigma_{1}$ at one point. Choose $s$ such that $c_{1}(s)$ is Poincaré dual to $\left(2-2\left(\operatorname{genus}\left(\Sigma_{1}\right)+1\right)\right)\left[\Sigma_{2}\right]$. Again we have $d=0$, so our construction gives an almost complex structure which extends across a ball containing the two circles in $Z$, and is compatible with a symplectic form outside that ball. Also, $\Sigma_{1}^{\prime}$ is a $J$-holomorphic curve which does not minimize genus.

Addendum [6 also allows us to carry out our construction on a 4-manifold which looks like $\mathbb{R}^{4}$ outside a compact set, to get a standard symplectic form at 
infinity. To make this more precise, let $W$ be a compact, oriented 4-manifold with $\partial W=S^{3}$. Let $\Sigma \subset W$ be a properly imbedded surface which uses up all the 3 -handles, with $\partial \Sigma$ unknotted in $S^{3}$. Let $[\Sigma]$ refer to the absolute class obtained by capping off $\partial \Sigma$ with a disk in $S^{3}$, and suppose that $[\Sigma] \cdot[\Sigma]>0$. Furthermore suppose there exists an integral lift $c$ of $w_{2}(W)$ such that $c \cdot[\Sigma]=$ $-2($ genus $(\Sigma)+1)+[\Sigma] \cdot[\Sigma]$. At the cost of increasing genus $(\Sigma)$, we can always find such a $c$.

Corollary 8 There exist a closed 2-form $\omega$ on $W$ which is symplectic on the complement of a collection of circles $Z \subset \operatorname{int}(W)$ and 0 along $Z$, an $\omega-$ compatible almost complex structure $J$ on $W-Z$, and a Riemannian metric $g$ on $W$ with respect to which $\omega$ is self-dual and transverse to 0 , such that:

(1) $[\omega]$ is Poincaré dual to $[\Sigma]$.

(2) $\Sigma^{\prime}$ is $J$-holomorphic, where $\Sigma^{\prime}$ is the connected sum of $\Sigma$ with a standard trivial torus in a ball.

(3) $\omega \mid \partial W=d \alpha_{0}$ for the standard contact form $\alpha_{0}$ on $S^{3}=\partial B^{4} \subset\left(\mathbb{R}^{4}, d x_{1} \wedge\right.$ $\left.d y_{1}+d x_{2} \wedge d y_{2}\right)$

\section{Proof Let}

(1) $A=\left(S^{2} \times S^{2}\right)-B^{4}$,

(2) $F$ be a properly embedded disk in $A$ normal to one of the $S^{2}$ 's,

(3) $X=W \cup_{S^{3}} A$,

(4) $\Sigma_{1}=\Sigma \cup F$, a closed surface with $\Sigma_{1} \cdot \Sigma_{1}=[\Sigma] \cdot[\Sigma]$,

(5) $\Sigma_{2}=S^{2} \times p \subset A$, and

(6) $\Sigma_{3}=p \times S^{2} \subset A$.

This gives the suitable input for Addendum [6] with the determinant of the intersection matrix equal to $-[\Sigma] \cdot[\Sigma]$ and with $s$ chosen so that $c_{1}(s) \cdot \Sigma_{2}=$ $c_{1}(s) \cdot \Sigma_{3}=2$. Then the output is standard near $\Sigma_{2} \cup \Sigma_{3}$, so we can restrict to $W$ to get standard behavior along $\partial W$.

Finally, there are certain special situations where we do not need to increase the genus of $\Sigma_{1}$. Let $\Sigma_{1}, \ldots, \Sigma_{k}$ be as in Addendum [6] above, and let $s$ be chosen so that, for $i=1, \ldots, k, c_{1}(s) \cdot \Sigma_{i}=2-2 g\left(\Sigma_{i}\right)+\Sigma_{i} \cdot \Sigma_{i}$.

Addendum 9 Suppose that we are in one of the following three cases:

(1) $k=2$, both surfaces are spheres, $\Sigma_{1} \cdot \Sigma_{1} \geq 2$ and $\Sigma_{2} \cdot \Sigma_{2} \geq 1$. 
(2) $\quad k=2, \operatorname{genus}\left(\Sigma_{1}\right)=0, \operatorname{genus}\left(\Sigma_{2}\right) \geq 1$ and $\Sigma_{i} \cdot \Sigma_{i} \geq 1$ for $i=1,2$.

(3) $k>2$, genus $\left(\Sigma_{1}\right)=0, \Sigma_{1} \cdot \Sigma_{1} \geq 1, \Sigma_{1} \cdot \Sigma_{2}=1$ and $\Sigma_{1} \cdot \Sigma_{i}=0$ for $i \geq 2$.

Then we have the same conclusions as in Addendum [6] except that $\Sigma_{1}$ is $J$ holomorphic rather than $\Sigma_{1}^{\prime}$.

Acknowledgements The authors would like to thank Yasha Eliashberg for numerous helpful suggestions and questions, András Stipsicz for help understanding homotopy classes of plane fields and Noah Goodman for some good ideas about making contact structures overtwisted. We would also like to thank the National Science Foundation for financial support under FRG grant number DMS-0244558; the first author's work was also supported by a CRM/ISM fellowship.

\section{The construction modulo details}

Henceforth all manifolds will be oriented, all symplectic structures on 4-manifolds will agree with the orientations of the manifolds, and all contact structures will be co-oriented. However we will work with both positive and negative contact structures.

Throughout this paper, if we refer to a triple $(\omega, J, \xi)$ (appropriately decorated with subscripts) on a pair $(W, \partial W)$, we mean that $W$ is a $4-$ manifold with boundary, $\omega$ is a closed 2 -form on $W$ which vanishes along a (possibly empty) collection of circles $Z$ and is symplectic on $W-Z, J$ is an almost complex structure on $W-Z$, and $\xi$ is a contact structure on $\partial W$. For such triples, $J$ will always be compatible with $\omega$, while $\xi$ will be compatible with $\omega$ and $J$ in the following sense: There should exist a Liouville vector field $V$ defined on a neighborhood of $\partial W$ and transverse to $\partial W$, inducing a contact form $\alpha=\imath_{V} \omega \mid \partial W$, with $\xi=\operatorname{ker} \alpha, J(\xi)=\xi$ and $J(V)=R_{\alpha}$ (the Reeb vector field for $\alpha$ ). Note that when $V$ points out of $W, \xi$ is positive (and $\partial W$ is said to be convex), and when $V$ points in, $\xi$ is negative (and $\partial W$ is said to be concave).

In this section we will lay out the construction and defer various details to later sections. First we give the construction starting with a single surface.

The construction (proof of Theorem 1) Split $X$ as $X=E \cup N$, where $E$ is a $B^{2}$-bundle neighborhood of $\Sigma$ and $N=X-\operatorname{int}(E)$. Let $Y=\partial N=-\partial E$, let $c=c_{1}(s)$ and let $g=\operatorname{genus}(\Sigma)$. 
Here is a very brief sketch of the construction of $\omega, Z$ and $J$ on $X$ such that $c_{1}(J)=c$. We will construct triples $\left(\omega_{E}, J_{E}, \xi_{E}\right)$ on $(E, \partial E)$ and $\left(\omega_{N}, J_{N}, \xi_{N}\right)$ on $(N, \partial N)$ with the following properties:

(1) $\omega_{E}$ and $J_{E}$ are defined on all of $E$.

(2) $\omega_{N}$ is defined on all of $N$ but vanishes along $Z \subset N$, while $J_{N}$ is defined on $N-Z$.

(3) $\xi_{E}$ is negative (as a contact structure on $\partial E$, hence positive on $Y$ ) and overtwisted.

(4) $\xi_{N}$ is positive and overtwisted.

(5) $\xi_{E}$ is homotopic as a plane field to $\xi_{N}$.

(6) $c_{1}\left(J_{N}\right)=c \mid N$ and $c_{1}\left(J_{E}\right)=c \mid E$.

Since $\xi_{E}$ and $\xi_{N}$ are both overtwisted, positive as contact structures on $Y$, and homotopic, we know 4 that they are isotopic as contact structures. This means that, after rescaling $\omega_{E}$ and perturbing $\omega_{E}$ and $J_{E}$ on a small collar neighborhood of $\partial E$, we can glue $\left(\omega_{E}, J_{E}\right)$ to $\left(\omega_{N}, J_{N}\right)$ to get $(\omega, J)$ on $X$. A little algebraic topology shows that $c_{1}(J)=c$.

Now we present the construction in more detail.

Choose integers $l_{1}, \ldots, l_{n} \in\{-1,+1\}$, with $l_{1}=-1$, so that $\sum_{i=1}^{n} l_{i}=d$; these will be the signs associated to the zero circles $Z_{1}, \ldots, Z_{n}$. (If $d<0$ a natural choice is $n=d$ and $l_{1}=\ldots=l_{d}=-1$ and if $d>0$ a natural choice is $n=d+2, l_{1}=-1$ and $l_{2}=\ldots=l_{d+2}=1$. However, any choice will work.)

First we establish a Morse function on $X$ with particular properties. Consider the obvious Morse function on $E$ with one 0 -handle, $2 g$-handles and one 2handle. Extend this to a Morse function on all of $X$ which has only $2-$ handles, 3 -handles and a single 4 -handle in $N$, and then turn this Morse function upside down. Introduce a cancelling $1-2-$ handle pair inside $N$. Label the $2-$ handles $H_{1}^{2}, \ldots, H_{p}^{2}, H_{p+1}^{2}$, where $H_{p+1}^{2}$ is the single 2-handle in $E$ and $H_{1}^{2}$ is the 2 handle from the cancelling 1-2 pair. Let $H_{1}^{1}$ be the 1 -handle cancelled by $H_{1}^{2}$. Slide $H_{p+1}^{2}$ over $H_{1}^{2}$ so that $H_{p+1}^{2}$ runs over $H_{1}^{1}$ once and then (possibly) over some other 1 -handles. Slide $H_{1}^{1}$ over these other 1 -handles so as to arrange that $H_{p+1}^{2}$ runs only over $H_{1}^{1}$. This gives a new Morse function which we will call $f$. Note that $f$ still respects the splitting $X=E \cup_{Y} N$ in the sense that we may take $Y$ to be a level set. (This is because we did not slide handles from $N$ over handles from $E$.)

For any given $t \in \mathbb{R}$, let $X_{t}=f^{-1}(-\infty, t]$ and let $Y_{t}=f^{-1}(t)=\partial X_{t}$. For future convenience, reparametrize $f$ so that $X_{k}$ is the union of the 0 - through 
$k$-handles, with $N=X_{1.5}$ and $Y=Y_{1.5}$. Also arrange that all the 1 -handles are in fact in $X_{0.9} \subset X_{1}$. (We will put the zero locus $Z$ into $f^{-1}[0.9,1]$.) Figure 1 illustrates this Morse function. Note that $f$ gives a cell decomposition to $X$, with the descending manifolds for each index $k$ critical point being the $k$-cells. Label the $k$-cells $C_{i}^{k}$ (corresponding to handles $H_{i}^{k}$ ). We will work with cellular cohomology and homology with respect to this cell decomposition, and represent cohomology classes by cellular cocycles. Since $X_{k}$ deformation retracts onto the $k$-skeleton of $X$, we will frequently represent classes in $H^{i}\left(X_{k} ; \mathbb{Z}\right)$ by cocycles on the $k$-skeleton of $X$. Because of the handle slides in the previous paragraph, we know that $\partial C_{p+1}^{2}=C_{1}^{1}$ in the cellular chain complex coming from $f$.

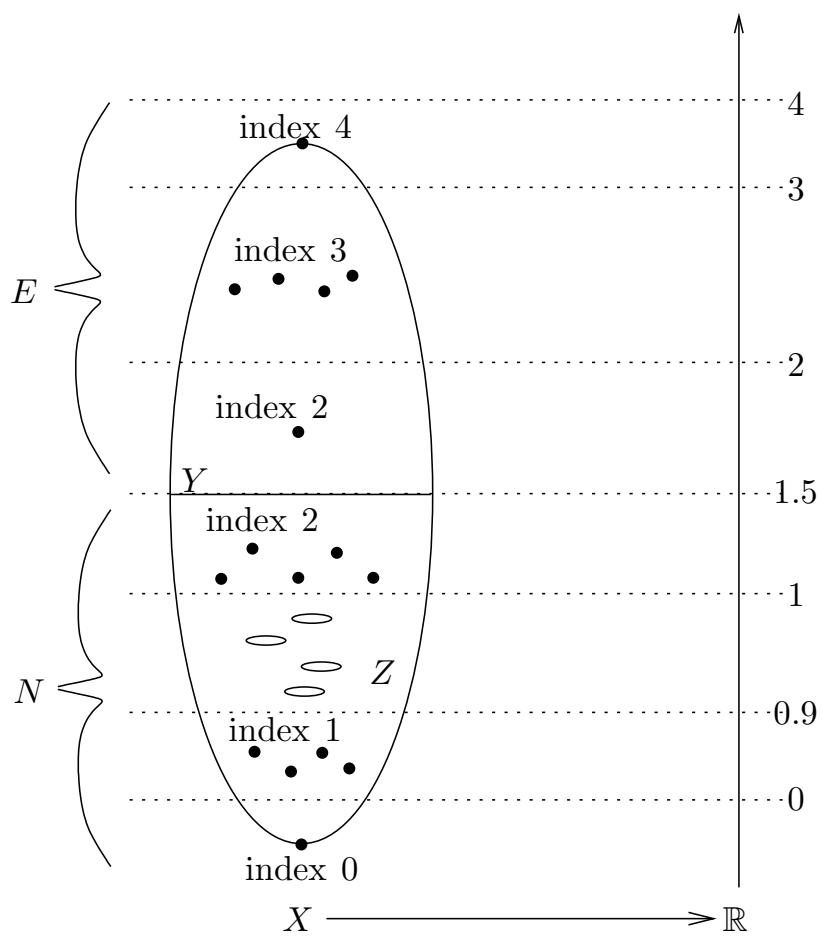

Figure 1: The Morse function $f: X \rightarrow \mathbb{R}$; dots are critical points, circles in $f^{-1}[0.9,1]$ are components of $Z$.

In Section 3 (Proposition 15), we construct the triple $\left(\omega_{E}, J_{E}, \xi_{E}\right)$. This is done by seeing $E$ as a neighborhood $E^{\prime}$ of $\Sigma^{\prime}$ together with two 2-handles. The triple is constructed first on $E^{\prime}$ as a standard symplectic neighborhood of a $J$-holomorphic curve $\Sigma^{\prime}$, with negative but tight contact boundary, and 
then the triple is extended over the two extra 2-handles required to make $E$ so that the boundary becomes overtwisted. Here we use a characterization [7] of neighborhoods of symplectic surfaces in terms of open books. Since $\Sigma^{\prime}$ is $J_{E^{-}}$ holomorphic we have $c_{1}\left(J_{E}\right)=c \mid E$. In Section 3 there are various perturbations of the Morse function inside $E$ (handle slides and introduction of cancelling pairs); after Section 3 we will abandon these perturbations and return to the original Morse function $f$.

Since $X_{0.9}$ is built from a 0 -handle and some 1 -handles, there is a more or less canonical construction of a triple $\left(\omega_{0.9}, J_{0.9}, \xi_{0.9}\right)$ on $\left(X_{0.9}, Y_{0.9}\right)$, with $\omega_{0.9}$ and $J_{0.9}$ defined everywhere and with $\xi_{0.9}$ positive and tight (see Proposition 10). In Section 4 (Proposition 20) we show how to extend this to a singular triple $\left(\omega_{1}, J_{1}, \xi_{1}\right)$ on $\left(X_{1}, Y_{1}\right)$, with $\omega_{1}$ vanishing on a union of circles $Z \subset f^{-1}[0.9,1]$ and $J_{1}$ defined on $X_{1}-Z$, so that $\xi_{1}$ is positive and overtwisted. In fact, $Z$ consists of one circle in each of $n$ levels between $Y_{0.9}$ and $Y_{1}$, in the following sense: Each component $Z_{i}$ of $Z$ arises in the product cobordism $f^{-1}[a(i), a(i+$ 1)] from $\left(Y_{a(i)}, \xi_{a(i)}\right)$ to $\left(Y_{a(i+1)}, \xi_{a(i+1)}\right)$ ( $i$ ranging from 1 to $n$ and $a(i)$ ranging from 0.9 to 1$)$, where each $\xi_{a(i)}$ is a positive contact structure and $\xi_{a(i+1)}$ differs from $\xi_{a(i)}$ by a (half) Lutz twist along a transverse unknot $U_{i} \subset\left(Y_{a(i)}, \xi_{a(i)}\right)$. The circle $Z_{i}$ is $0.5 \times U_{i}$ after identifying $f^{-1}[a(i), a(i+1)]$ with $[0,1] \times Y_{a(i)}$. Also, $\omega_{1} \mid f^{-1}[a(i), a(i+1)]$ is a standard symplectification of $\xi_{a(i)}$ outside $[0,1] \times$ $T_{i}$ for some solid torus neighborhood $T_{i}$ of $U_{i}$. Here we also construct a metric $g$ such that $\omega_{1}$ is $g$-self-dual and transverse to the zero section of $\Lambda_{+}^{2}$. (Outside a small neighborhood of $Z, g$ is determined by $\omega_{1}$ and $J_{1}$ in the usual way, but the metric determined by $\omega_{1}$ and $J_{1}$ develops a singularity along $Z$ which we remove by suitably rescaling.)

A homologically trivial transverse knot $K$ comes with an integer invariant, the self-linking number $\operatorname{lk}(K)$. We will use the fact that any positive contact manifold has transverse unknots with $\mathrm{lk}=-1$, and that if the contact structure is overtwisted we can also find transverse unknots with $\mathrm{lk}=+1$ (lemma 12). Each $U_{i}$ is chosen so that $\operatorname{lk}\left(U_{i}\right)=l_{i}$ (hence the requirement that $l_{1}=-1$ ). In Section 5] (lemma 25) we show that, if $B_{i}$ is a 4-ball neighborhood of a disk bounded by $Z_{i}$, then the obstruction to extending $J_{1}$ across $B_{i}$ is exactly $\operatorname{lk}\left(U_{i}\right)=l_{i}$.

At this point fix a trivialization $\tau$ of $\xi_{1}$ (possible because $c_{1}\left(\xi_{1}\right)=0$ ). Let $L=K_{1} \cup \ldots \cup K_{p} \cup K_{p+1}$ be the link of attaching circles for all the 2 -handles $H_{1}^{2} \cup \ldots \cup H_{p}^{2} \cup H_{p+1}^{2}$ of $X$ as seen in $\left(Y_{1}, \xi_{1}\right)$. Each handle $H_{i}$ is to be attached with some framing $F_{i}$ of $K_{i}$. Because $\xi_{1}$ is overtwisted, we may isotope $L$ to be Legendrian with $\operatorname{tb}\left(K_{i}\right)-1=F_{i}$ (see lemma 11). Thus (Proposition 10, see [18]), we can extend $\left(\omega_{1}, J_{1}, \xi_{1}\right)$ to a triple on $\left(X_{2}, Y_{2}\right)$ which we will label 
$\left(\omega_{2}^{\prime}, J_{2}^{\prime}, \xi_{2}^{\prime}\right)$. (We will soon change some choices and replace this triple with a better one, which we will call $\left(\omega_{2}, J_{2}, \xi_{2}\right)$.)

With respect to the trivialization $\tau$, each (oriented) Legendrian knot $K_{i}$ has a rotation number $\operatorname{rot}\left(K_{i}\right)$ (the winding number of $T K_{i}$ in $\xi_{1} \mid K_{i}$ relative to $\tau)$. Let $x^{\prime}$ be the cochain whose value on a 2 -cell $C_{i}^{2}$ is exactly $\operatorname{rot}\left(K_{i}\right)$ for the corresponding $K_{i}$. As a cochain on the 2-skeleton of $X, x^{\prime}$ is trivially a cocycle; in Section [6 (lemma 27) we show that $x^{\prime}$ represents $c_{1}\left(J_{2}^{\prime}\right) \in H^{2}\left(X_{2} ; \mathbb{Z}\right)$ (since $X_{2}$ deformation retracts onto the 2 -skeleton of $X$ ). We would like to have $c_{1}\left(J_{2}^{\prime}\right)=c \mid X_{2}$, but this is probably not the case $\left(x^{\prime}\right.$ is probably not even a cocycle on $X)$.

Represent $c$ by a cocycle $x$ on $X$ which is congruent mod 2 to $x^{\prime}$. (For any representative $x$ of $c$, since both $c_{1}\left(J_{2}^{\prime}\right)$ and $c \mid X_{2}$ reduce mod 2 to $w_{2}\left(X_{2}\right), x-x^{\prime}$ is congruent mod 2 to $\delta y$ for some 1 -cochain $y$. Thus we can replace $x$ with $x-\delta y$ if necessary.) In Section 2 (lemma 11) we show that we can isotope any Legendrian knot $K$ in an overtwisted contact structure to a new Legendrian knot, without changing $\operatorname{tb}(K)$, so as to change $\operatorname{rot}(K)$ by any even number. Thus we can arrange that $\operatorname{rot}\left(K_{i}\right)=x\left(C_{i}^{2}\right)$ for each 2-cell $C_{i}^{2}$. Now discard $\left(\omega_{2}^{\prime}, J_{2}^{\prime}, \xi_{2}^{\prime}\right)$ and use the new $K_{i}$ 's as attaching circles. Attach $H_{1}^{2}, \ldots, H_{p}^{2}$ along $K_{1}, \ldots, K_{p}$ to get $\left(\omega_{N}, J_{N}, \xi_{N}\right)$ on $(N, Y)=\left(X_{1.5}, Y_{1.5}\right)$. Then attach $H_{p+1}^{2}$ along $K_{p+1}$ to extend $\left(\omega_{N}, J_{N}, \xi_{N}\right)$ to $\left(\omega_{2}, J_{2}, \xi_{2}\right)$ on $\left(X_{2}, Y_{2}\right)$. Thus (Proposition [27) $c_{1}\left(J_{2}\right)=[x]=c \mid X_{2}$ and $c_{1}\left(J_{N}\right)=[x]|N=c| N$. In the end we will only use $\left(\omega_{N}, J_{N}, \xi_{N}\right)$, but we need $J_{2}$ to show that $\xi_{N}$ is homotopic to $\xi_{E}$. Here we use Proposition 10 again to know that our construction extends over the 2-handles; this proposition also tells us that $\omega_{N}$ is exact.

To be sure that $\xi_{N}$ is overtwisted we should arrange that there is some fixed overtwisted disk which is missed by all the attaching circles for the 2 -handles. This is possible because, as a result of a Lutz twist, we have a circles' worth of overtwisted disks, whereas to adjust tb and rot for the attaching circles we only need a neighborhood of a single overtwisted disk.

We show in Section [6 (lemma 28) that, precisely because $x$ is a cocycle on all of $X$, the almost complex structure $J_{2}$ will extend across the 3 -cells of $X$, and hence will extend to $J_{3}$ on $X_{3}$. (Here we abandon the symplectic form and contact structure.) We still have $c_{1}\left(J_{3}\right)=c$. Recall the 4 -ball neighborhoods $B_{i}$ of disks bounded by the circles $Z_{i}$. Let $B_{*}=f^{-1}[3, \infty)$ be the single $4-$ handle for $X$. We can think of $J_{3}$ as defined on $X-\left(B_{1} \cup \ldots \cup B_{n} \cup B_{*}\right)$. It is known [1] (and explained in Section 5, lemma 24) that the total obstruction to extending an almost complex structure $J$ defined on the complement of some balls in a closed $4-$ manifold $X$ over those balls is precisely $\left(c_{1}(J)^{2}-3 \sigma(X)-\right.$ 
$2 \chi(X)) / 4$. But we have arranged that the sum of the obstructions to extending $J_{3}$ over $B_{1}, \ldots, B_{n}$ is already $d=\left(c_{1}\left(J_{3}\right)^{2}-3 \sigma(X)-2 \chi(X)\right) / 4$, and thus the obstruction to extending across $B_{*}$ is 0 . Therefore $J_{3}$ extends over the 4 -handle to an almost complex structure $J_{*}$ on $X-Z$.

Now compare $J_{*} \mid E$ and $J_{E}$. We know that $c_{1}\left(J_{*} \mid E\right)=c \mid E=c_{1}\left(J_{E}\right)$, that $H^{2}(E ; \mathbb{Z})$ has no 2 -torsion, and that $E$ deformation retracts onto a 2-complex (coming from the dual Morse function $-f$ ). In Section 7 (lemma 29), we show that this implies that $J_{E}$ is homotopic to $J_{*} \mid E$. Therefore $\xi_{E}$ is homotopic to $\xi_{N}$ (this also follows from [9]) and we can glue $\left(E, \omega_{E}, J_{E}\right)$ to $\left(N, \omega_{N}, J_{N}\right)$ as described above to get $(X, \omega, J)$.

Now we have $J$ on $X-Z$ with $c_{1}(J \mid N)=c \mid N$ and $c_{1}\left(J_{E}\right)=c \mid E$. This implies that $c_{1}(J)=c$ because, in the cohomology Mayer-Vietoris sequence for $X=E \cup N$, the map $H^{1}(E) \oplus H^{1}(N) \rightarrow H^{1}(E \cap N)$ is surjective, so that the map $H^{2}(X) \rightarrow H^{2}(E) \oplus H^{2}(N)$ is injective. (The precise topology of $E$ and $Y=E \cap N$ is important here.)

Now suppose that our construction produced the $\operatorname{spin}^{\mathbb{C}}$ structure $s_{0}$ when instead we wanted $s_{1}=s$. We know that $s_{1}$ is the result of acting on $s_{0}$ by some class $a \in H^{2}(X ; \mathbb{Z})$ of order 2 . Our construction above was based on a choice of cocycle representative $x$ for $c$. In Section 7 (Proposition 30), we show that if, instead of $x_{0}=x$, we had used $x_{1}=x+2 z$ for a special cocycle representative $z$ of $a$, then we would have produced the desired $\operatorname{spin}^{\mathbb{C}}$ structure $s_{1}$.

The metric $g$ is constructed first on $X_{1}$ as mentioned earlier; on the rest of $X, g$ is determined by $\omega$ and $J$. In dimension 4 , if a metric $g$ is given by a symplectic form $\omega$ and a compatible almost complex structure, then $\omega$ is automatically $g$-self-dual.

Finally we can rescale $\omega$ so that $\int_{\Sigma} \omega=[\Sigma] \cdot[\Sigma]$. Let $\sigma$ be the Poincaré dual of $[\Sigma]$ in $H^{2}(X ; \mathbb{R})$. Then we know that $[\omega]|E=\sigma| E$. Also, because $\omega$ is exact on $N$, we know that $[\omega]|N=\sigma| N$. As with $c_{1}(J)$, the Mayer-Vietoris sequence then gives that $[\omega]=\sigma$.

Proof of Addendum [ 6 Now let $E$ be a regular neighborhood of $\Sigma_{1} \cup \ldots \cup \Sigma_{n}$ (a plumbing of $B^{2}$-bundles given by a plumbing graph $G$, vertices corresponding to surfaces and edges corresponding to intersections), and let $N=$ $X-\operatorname{int}(E)$. Recall that $Q$ is the intersection form for $E$. The construction is essentially the same except for the following changes:

The Morse function we choose on $X$ begins with a standard Morse function on $E$ with one 0 -handle for each edge in $G$, one 0 -handle for each vertex, 
one 1-handle for each incidence between an edge and a vertex, and 2 genus $\left(\Sigma_{i}\right)$ 1-handles and one 2-handle for each surface $\Sigma_{i}$ (see [6]). (Of course we can cancel many 0-1-handle pairs, but then the picture becomes less canonical.) Extend to $X$ and turn upside down, as before. Now, for each $\Sigma_{i}$, introduce a cancelling 1-2-handle pair and slide some handles so that the attaching circle for the 2-handle coming from $\Sigma_{i}$ runs exactly once over exactly one 1-handle, as we did for the single 2 -handle coming from $E$ in the preceding proof.

Given this handlebody decomposition, the construction of $\left(\omega_{N}, J_{N}, \xi_{N}\right)$ is unchanged. In Section 3 (Proposition [16) we show how to adjust the construction of $\left(\omega_{E}, J_{E}, \xi_{E}\right)$ to handle the case where $E$ is a plumbing of many disk bundles, again using a characterization of neighborhoods of configurations of symplectic surfaces in [6].

To see that our construction gives the correct $c_{1}(J)$, again we use the fact that the map $H^{1}(E) \rightarrow H^{1}(Y)$ is surjective for the plumbings that we are dealing with. (Here is where we need that $\operatorname{det}(Q) \neq 0$.) To see that we can achieve the correct $\operatorname{spin}^{\mathbb{C}}$ structure even in the presence of 2 -torsion, we use the same argument from the main proof, since we have arranged that each 2-handle from $E$ runs once over one 1-handle.

Proposition [16] also arranges that $\int_{\Sigma_{i}} \omega_{E}=\Sigma_{1} \cdot \Sigma_{i}+\ldots+\Sigma_{k} \cdot \Sigma_{i}$ for each $\Sigma_{i}$. Thus, if we let $\sigma$ be the Poincaré dual of $\left[\Sigma_{1}\right]+\ldots+\left[\Sigma_{k}\right]$, we see again that $[\omega]|E=\sigma| E$ and $[\omega]|N=\sigma| N$, so that $[\omega]=\sigma$.

Proof of Addendum 9 In Section 5.4 of 8, Goodman investigates conditions under which concave boundaries of configurations of symplectic surfaces have overtwisted boundaries. The techniques there show precisely that the configurations in Addendum 9 have symplectic neighborhoods with concave overtwisted boundary. We use this structure for $\left(\omega_{E}, J_{E}, \xi_{E}\right)$ and the rest of the construction is as before.

\section{Brief symplectic and contact prerequisites}

Suppose that $W^{4}$ is a 4 -manifold with boundary $\partial W=M^{3}$, and suppose that a triple $(\omega, J, \xi)$ has been constructed on $(W, M)$. Let $K$ be a Legendrian knot in $(M, \xi)$. $K$ comes with a natural contact framing $\operatorname{tb}(K)$ (standing for "Thurston-Bennequin"), given by any vector field in $\xi \mid K$ orthogonal to $K$. Let $W^{\prime}$ be the result of attaching a 2 -handle $H$ along $K$ with framing $\operatorname{tb}(K)-1$. Alternatively, suppose that $W^{\prime}$ is the result of attaching a 1-handle $H$ to $W$, 
with no constraints on the attaching map, but with the assumption that $\xi$ is positive. In either case, we have the following well known result:

Proposition 10 (Weinstein 18, Eliashberg [5]) The triple $(\omega, J, \xi)$ extends to $\left(\omega^{\prime}, J^{\prime}, \xi^{\prime}\right)$ on $\left(W^{\prime}, M^{\prime}=\partial W^{\prime}\right)$, with $\omega^{\prime}$ symplectic on all of $H$ and $J^{\prime}$ defined on all of $H$. Furthermore $\xi=\xi^{\prime}$ outside the surgery that changes $M$ to $M^{\prime}$. If $\omega$ was exact then $\omega^{\prime}$ is also exact.

As originally presented in [18 and [5], this applied only to the case where $\omega$ was symplectic everywhere and $\xi$ was positive. As long as the singularities of $\omega$ stay in $\operatorname{int}(W)$, extending to singular symplectic forms is trivial. When $\xi$ is negative and $H$ is a 2 -handle, we should turn the original model handles of [18] and [5] upside down. This is discussed in [2].

In 2] it is also shown that, in the 2-handle case, the surgery that turns $(M, \xi)$ into $\left(M^{\prime}, \xi^{\prime}\right)$ is uniquely determined up to isotopy fixed outside a neighborhood of $K$ by the property that it preserves tightness near $K$. This unique contact surgery is called tb -1 surgery along $K$. There is also a uniquely determined tb +1 surgery, which is precisely what we see if we attach a 2 -handle as in the proposition to a negative contact boundary, but reverse orientations so that we see our contact structure as positive.

When an oriented Legendrian knot $K$ bounds a surface $F \subset M$, there is a welldefined integer $\operatorname{rot}(K)$, the rotation number of $K$, given by trivializing $\xi$ over $F$ and counting the winding number of $T K$ relative to this trivialization. If $c_{1}(\xi)=0$, then we can pick a global trivialization of $\xi$ which works for all $F$ 's. Then, even for homologically nontrivial knots $K$, we get a well-defined rotation number relative to this trivialization, which we again call $\operatorname{rot}(K)$. (Note that $\operatorname{rot}(-K)=-\operatorname{rot}(K)$.)

Lemma 11 In the above situation, suppose that $a, b$ are integers with $a+b \equiv$ $\operatorname{tb}(K)+\operatorname{rot}(K) \bmod 2$. If $\xi$ is overtwisted then we may smoothly isotope $K$ to another Legendrian knot $K^{\prime}$ such that $\operatorname{tb}\left(K^{\prime}\right)=a$ and $\operatorname{rot}\left(K^{\prime}\right)=b$.

Proof In a standard contact chart we can perform a connected sum between two Legendrian knots $K_{0}$ and $K_{1}$ as illustrated in Figure 2 (which is a standard "front projection" onto the $y z$ coordinate plane, where the contact structure is $\operatorname{ker}(d z+x d y))$. Locally we can assume that our trivialization of $\xi$ is the vector field $\frac{\partial}{\partial x}$. Then $\operatorname{tb}\left(K_{0} \# K_{1}\right)=\operatorname{tb}\left(K_{0}\right)+\operatorname{tb}\left(K_{1}\right)+1$ and $\operatorname{rot}\left(K_{0} \# K_{1}\right)=$ $\operatorname{rot}\left(K_{0}\right)+\operatorname{rot}\left(K_{1}\right)$. In any positive contact 3 -manifold, there is a Legendrian unknot $K_{-2,1}$ with $\operatorname{tb}\left(K_{-2,1}\right)=-2$ and $\operatorname{rot}\left(K_{-2,1}\right)= \pm 1$. For example, see 
Figure 3. In any positive, overtwisted contact $3-$ manifold there is a Legendrian unknot $K_{0,1}$ with $\operatorname{tb}\left(K_{0,1}\right)=0$ and $\operatorname{rot}\left(K_{0,1}\right)= \pm 1$. (Consider the contact structure $\xi_{0}=\operatorname{ker}\left(\cos \left(r^{2}\right) d z+\sin \left(r^{2}\right) d \theta\right)$ on $\mathbb{R}^{3}$ with cylindrical coordinates $(r, \theta, z)$, and let $D$ be the overtwisted disk $\left\{r^{2} \leq \pi, z=0\right\}$. In every overtwisted contact 3-manifold one can find a ball contactomorphic to a neighborhood of $D$ with this contact structure. Let $K_{0,1}$ be the preimage of $\partial D$ under this contactomorphism. One can check explicitly that $\operatorname{tb}(\partial D)=0$ and $\operatorname{rot}(\partial D)=$ \pm 1 .) We construct $K^{\prime}$ as the connected sum of $K$ with some number of copies of $K_{-2,1}$ and $K_{0,1}$ so as to arrange that $\operatorname{tb}\left(K^{\prime}\right)=a$ and $\operatorname{rot}\left(K^{\prime}\right)=b$. Of course $K^{\prime}$ is smoothly isotopic to $K$.

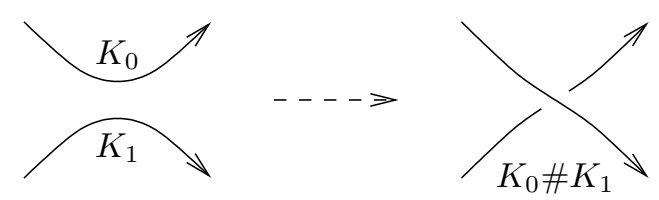

Figure 2: Connected sum of Legendrian knots

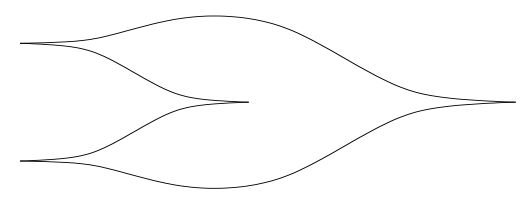

Figure 3: Knot with $(\mathrm{tb}, \mathrm{rot})=(-2, \pm 1)$

Now let $K$ be a homologically trivial transverse knot in $(M, \xi)$. There is an integer invariant of $K$ known as the self-linking number $\operatorname{lk}(K)$ given by trivializing $\xi$ over a surface $F$ bounded by $K$ and seeing this trivialization restricted to $K$ as a framing of $K$. Again consider the contact structure $\xi_{0}=\operatorname{ker}\left(\cos \left(r^{2}\right) d z+\sin \left(r^{2}\right) d \theta\right)$ on $\mathbb{R}^{3}$. Every contact 3 -manifold has charts contactomorphic to small neighborhoods of 0 in $\left(\mathbb{R}^{3}, \xi_{0}\right)$, and every overtwisted manifold has charts contactomorphic to small neighborhoods of $D=\left\{r^{2} \leq \pi\right\}$. Consider the circles $K_{R}=\{r=R\}$. The following can be easily checked:

Lemma 12 For small positive $\epsilon, K_{\epsilon}$ is transverse with $\operatorname{lk}\left(K_{\epsilon}\right)=-1$, and $K_{\sqrt{\pi}+\epsilon}$ is transverse with $\operatorname{lk}\left(K_{\sqrt{\pi}+\epsilon}\right)=+1$. Thus every contact manifold has knots with self-linking number -1 , and every overtwisted contact manifold has knots with self-linking number +1 . 


\section{Constructing $\left(\omega_{E}, J_{E}, \xi_{E}\right)$}

As a warmup and for the sake of completeness, we prove the following:

Lemma 13 Given any class $\alpha \in H_{2}(X ; \mathbb{Z})$, there exists an integer $g(\alpha)$ with the following property: For any $g \geq g(\alpha)$, there is a genus $g$ surface $\Sigma$ with $[\Sigma]=\alpha$ which uses up all the 3 -handles.

Proof Choose some embedded surface $\Sigma$ representing $\alpha$. Let $g=\operatorname{genus}(\Sigma)$ and let $W$ be a $B^{2}$-neighborhood of $\Sigma$. There exists a handlebody decomposition of $X$ with one 0 -handle, $2 g$-handles and one 2 -handle, forming $W$, together with $l$ more 1 -handles, some more 2 -handles, some 3 -handles and a 4 -handle. Let $H$ be the single 2 -handle in $W$; Figure 4 is a picture of $W$. (The framing for $H$ is $\alpha \cdot \alpha$.)

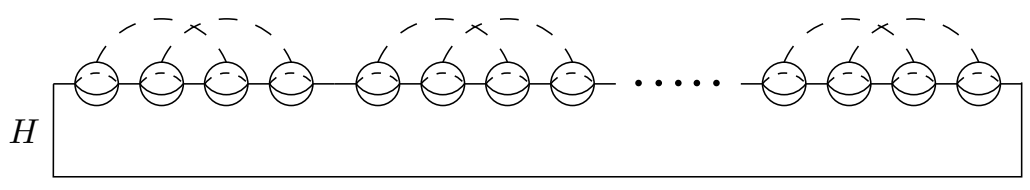

Figure 4: The initial handlebody decomposition for $W$, with one 2-handle $H$

Let $g(\alpha)=g+l$. Given $g^{\prime}=g(\alpha)+k \geq g(\alpha)$, introduce $k$ cancelling pairs of 1 - and 2-handles, so that now we have $q=l+k$ extra 1 -handles.

Let $A_{1}, \ldots, A_{q}$ be the extra 1 -handles and introduce $q$ more cancelling 12-handle pairs, with the 1 -handles labelled $B_{1}, \ldots, B_{q}$ and the respective 2handles labelled $C_{1}, \ldots, C_{q}$. Let $W^{\prime}=W \cup\left(A_{1} \cup \ldots \cup A_{q}\right)=W \cup\left(A_{1} \cup \ldots A_{q} \cup\right.$ $\left.B_{1} \cup \ldots \cup B_{q} \cup C_{1} \ldots \cup C_{q}\right)$. Now, for $i=1$ up to $q$, slide $H$ over $A_{i}$ then over $C_{i}$ twice, as in Figure 5, to get Figure 6. (We have suppressed framings in the figures, but the $C_{i}$ 's should be 0 -framed so that, after sliding, the framing on $H$ is still $\alpha \cdot \alpha$.)

With this new handlebody decomposition let $W^{\prime \prime}=W^{\prime}-\left(C_{1} \cup \ldots \cup C_{q}\right)$, and note that $W^{\prime \prime}$ is a neighborhood of a surface $\Sigma^{\prime}$ of genus $g+q=g(\alpha)+k=g^{\prime}$. The remainder of $X$ is built with $C_{1}, \ldots, C_{q}$ and some more 2-handles, 3 handles and a 4 -handle. Thus $X-W^{\prime \prime}$ has a handlebody decomposition with only $0-, 1-$ and 2 -handles.

To see that $\left[\Sigma^{\prime}\right]=[\Sigma]$, note that we slid $H$ over each $C_{i}$ once in a positive direction and once in a negative direction. 

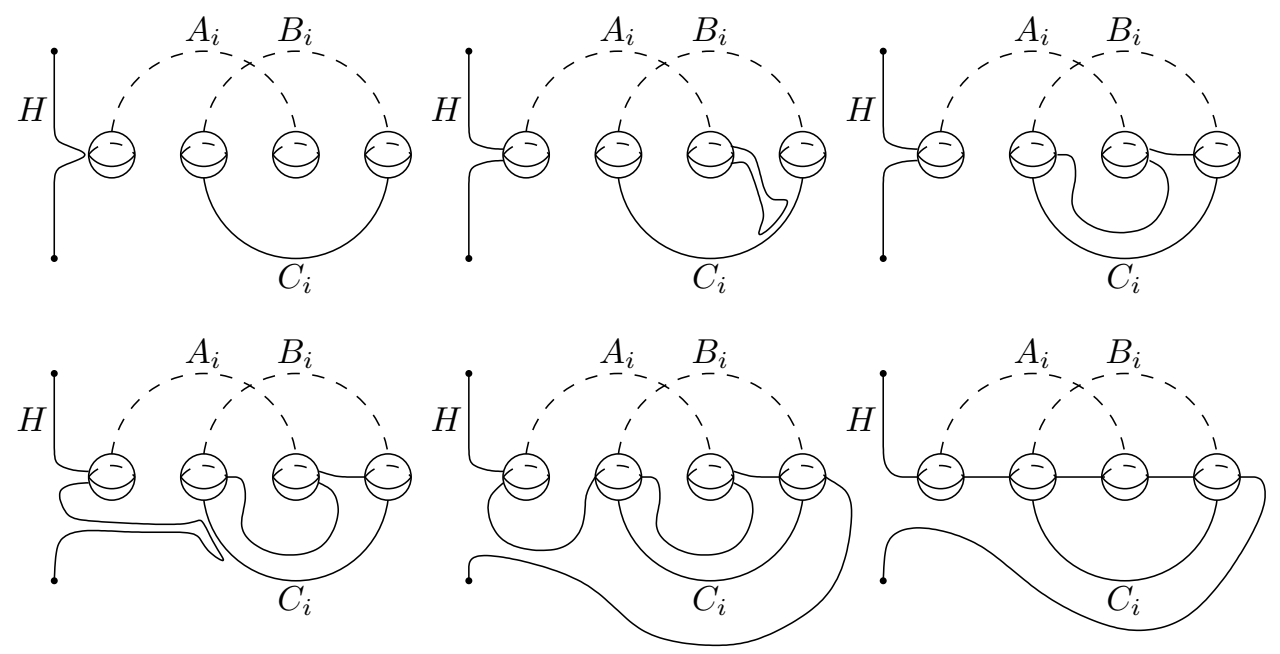

Figure 5: Sliding $H$ over a 1 -handle then twice over a 2 -handle

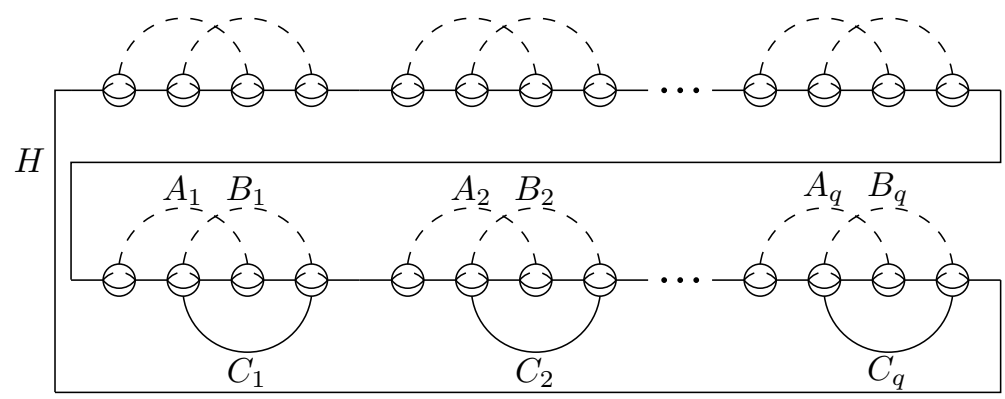

Figure 6: Handlebody decomposition of $W^{\prime}$

Now we return to the notation in the introduction: $\Sigma$ is a given surface of genus $g$ representing a class $\alpha \in H_{2}(X ; \mathbb{Z})$, with $B^{2}$-neighborhood $E$. Let $m=\alpha \cdot \alpha>0$. The Morse function $-f: X \rightarrow \mathbb{R}$ restricts to the obvious Morse function on $E$ with one 0 -handle, $2 g$-handles and one 2-handle, which we will label $H$ as in the proof of the preceding lemma. First introduce $m-1$ cancelling 1 -2-handle pairs inside $E$ (each new 2-handle framed +1 ) and slide $H$ over all the new $2-$ handles so that $E$ gets a handlebody decomposition with one 0-handle, $(2 g+m-1)$ 1-handles and $m$ 2-handles attached as in Figure 7 Now the framing on $H$ will also be +1 .

Next introduce a 1 -handle $A$ cancelled by a 2 -handle $B$ and a 1 -handle $C$ cancelled by a 2 -handle $D$, inside $E$. As in the proof of the preceding lemma, 


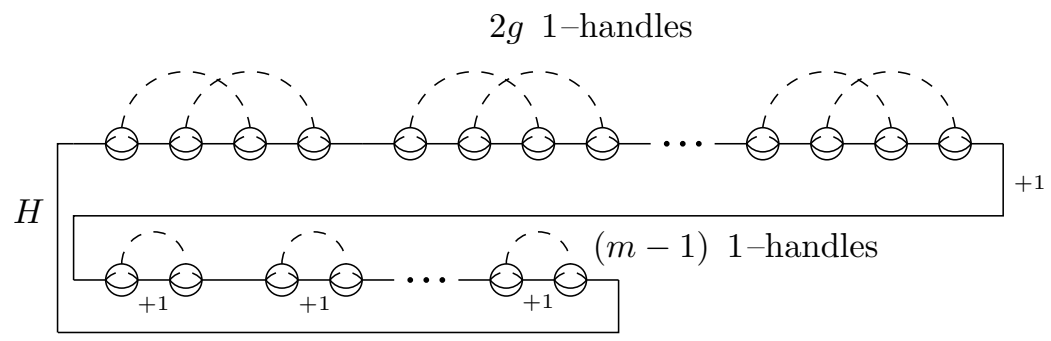

Figure 7: Handlebody decomposition of $E$

as in Figure [5, slide $H$ over $A$ and then over $D$ twice, to get Figure 8 . To get the right picture, the framing of $D$ should be 0 but the framing of $B$ can be anything so we have left it unlabelled in the figure. With respect to this

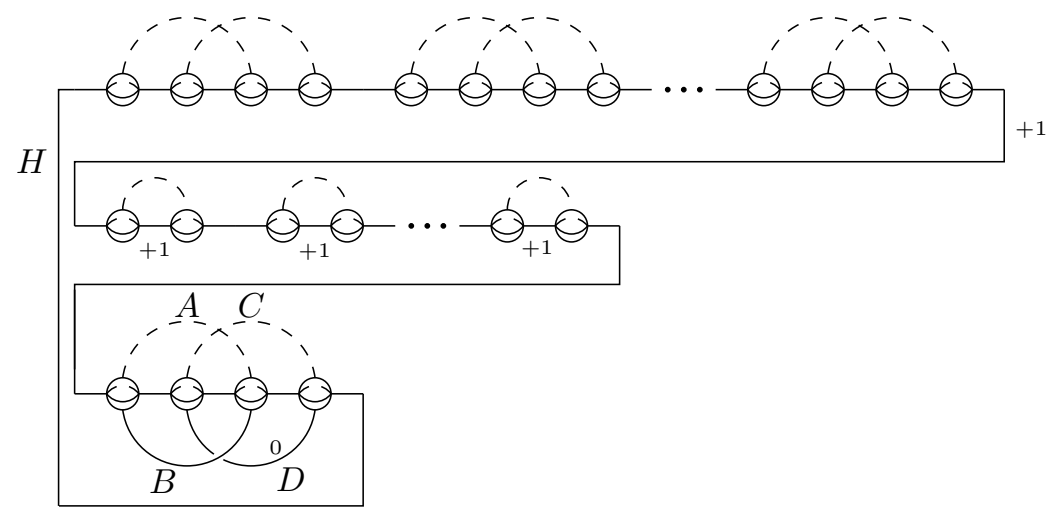

Figure 8: Another handlebody decomposition of $E$

new handlebody decomposition, let $E^{\prime}=E-(B \cup D)$. Note that $E^{\prime}$ is a neighborhood of a surface $\Sigma^{\prime}$ of genus $g^{\prime}=g+1$ formed as the connected sum of $\Sigma$ with a standard torus in a 4 -ball neighborhood of a point in $\Sigma$.

The first important point is that, if we attach $D$ to $E^{\prime}$ then we can slide $H$ back off of $D$ (twice) and over $A$ and then cancel $C$ and $D$. Therefore $E^{\prime} \cup D=E \curvearrowleft\left(S^{1} \times B^{3}\right)$, where the $S^{1} \times B^{3}$ summand comes precisely from $A$. Thus $\partial\left(E^{\prime} \cup D\right)=\partial E \#\left(S^{1} \times S^{2}\right)$, and $B$ is to be attached along any circle isotopic to $S^{1} \times\{p\}$ in the $S^{1} \times S^{2}$ summand.

The second important point is an understanding of the framings of the cancelling 2-handles $B$ and $D$. When constructing $E^{\prime}$ there is a natural open book decomposition of $\#^{\left(2 g^{\prime}+m-1\right)}\left(S^{1} \times S^{2}\right.$ ) (the boundary of the 0 -handle and the 1 -handles) with page an $m$-punctured genus $g^{\prime}$ surface and trivial 
monodromy. The construction of $E^{\prime}$ is completed by attaching $m 2$-handles along the binding, each with framing +1 (relative to the page), so that $\partial E^{\prime}$ inherits a natural open book decomposition. The page can still be seen clearly in Figure 8 as the disk bounded by the attaching circles for the 2-handles (not including $B$ and $D$ ) together with 2-dimensional 1-handles embedded in the 4-dimensional 1-handles. The attaching circles for $B$ and $D$ can be isotoped to lie on this page. To make the sliding of $H$ work right, we needed $D$ to be attached with framing 0 measured relative to this page.

To put a symplectic structure on $E$ with concave, overtwisted boundary, we will need the following:

Lemma 14 Given any negative contact structure $\xi$ on a $3-$ manifold $M^{3}$ and any Legendrian knot $K$ which transversely intersects a 2 -sphere $S$ in $M$ at one point, $\operatorname{tb}(K)-1$ surgery along $K$ leads to an overtwisted contact structure.

Proof First reverse the orientation of $M$. Then $\xi$ is a positive contact structure and we need to show that $\operatorname{tb}(K)+1$ surgery along $K$ leads to an overtwisted contact structure. We will show that, after surgery, $S$ becomes a disk with Legendrian boundary $K^{\prime}$ (the dual circle to $K$ ) with $\operatorname{tb}\left(K^{\prime}\right)=+1$, which is not possible in a positive tight contact structure.

Let $T_{0}=D^{2} \times S^{1}$ be a solid torus with coordinates $(r, \mu, \lambda)$, where $(r, \mu)$ are polar coordinates on $D^{2}$ and $D^{2}=\{r \leq 1\}$. Every Legendrian knot has a neighborhood which is contactomorphic to $T_{0}$ with a certain standard contact structure $\xi_{0}$ with the property that $\partial T_{0}=S^{1} \times S^{1}$ is a convex surface with dividing set $\Gamma_{0}=\{r=1, \mu \in\{0, \pi\}\}$ (see [2], for example). Here $K_{0}=\{r=0\}$ is Legendrian and $\operatorname{tb}\left(K_{0}\right)$ is the 0 -framing coming from our splitting of $T_{0}$ as $D^{2} \times S^{1}$. To perform surgery along a Legendrian knot $K \subset(M, \xi)$, find a neighborhood $T$ of $K$ contactomorphic to $\left(T_{0}, \xi_{0}\right)$. Then $M-\operatorname{int}(T)$ has convex torus boundary with dividing set $\Gamma$ equal to two parallel longitudes. Now glue $\left(T_{0}, \xi_{0}\right)$ back in via any diffeomorphism $\phi: \partial T_{0} \rightarrow \partial T$ which takes $\Gamma_{0}$ to $\Gamma$. Identifying $\partial T$ with $\partial T_{0}$ via the original contactomorphism between $(T, \xi)$ and $\left(T_{0}, \xi_{0}\right)$, we think of $\phi$ as an automorphism of $\partial T_{0}$ taking $\Gamma_{0}$ to $\Gamma_{0}$, and hence we think of $\phi \in S L(2, \mathbb{Z})$. Legendrian tb +1 surgery corresponds to $\phi=\left(\begin{array}{ll}1 & 0 \\ 1 & 1\end{array}\right)$, using the basis $(\mu, \lambda)$.

Recall our 2-sphere $S$ : Note that $S \cap \partial T$ is a meridian, or, after identifying $T$ with $T_{0}$ and lifting to the universal cover, the line spanned by $v=(1,0)^{T}$. Then $\phi^{-1}(v)=(1,-1)^{T}$. In other words, after surgery, $D=S-\operatorname{int}(T)$ is 
a disk which meets $\partial T_{0}$ in a longitude representing the framing $\operatorname{tb}\left(K_{0}\right)-1$. Extend $D$ in to $T_{0}$ by the obvious annulus so that $\partial D=K_{0}=K^{\prime}$. Then the (topological) canonical zero-framing of $K^{\prime}$ given by $D$ is $\operatorname{tb}\left(K^{\prime}\right)-1$, hence $\operatorname{tb}\left(K^{\prime}\right)=+1$.

Proposition 15 There exists a triple $\left(\omega_{E}, J_{E}, \xi_{E}\right)$ on $(E, \partial E)$ such that $\xi_{E}$ is negative and overtwisted, $\Sigma^{\prime}$ is $J_{E}$-holomorphic and $c_{1}\left(J_{E}\right)=c \mid E$.

Proof Since $E^{\prime}$ is a neighborhood of a surface $\Sigma^{\prime}$ with positive self-intersection, $E^{\prime}$ has a symplectic structure $\omega_{E^{\prime}}$ with concave boundary (by Theorem 1.1 in [7]), in which $\Sigma^{\prime}$ is symplectic. We can find a compatible almost complex structure $J_{E^{\prime}}$ with respect to which $\Sigma^{\prime}$ is holomorphic, and thus we get our triple $\left(\omega_{E^{\prime}}, J_{E^{\prime}}, \xi_{E^{\prime}}\right)$, with $\xi_{E^{\prime}}$ negative. Theorem 1.1 in [7] also says that $\xi_{E^{\prime}}$ is supported by the open book on $\partial E^{\prime}$ described above. (See Section 2 of [6] and Section 1 of [7] for background on the important relationship between contact structures and open books established by Giroux.) Let $K_{D}$ be the attaching circle for the 2 -handle $D$; we noted earlier that $K_{D}$ lies in a page of the open book. Since $K_{D}$ is also homologically nontrivial in that page, we may assume that $K_{D}$ is Legendrian, with $\operatorname{tb}\left(K_{D}\right)$ equal to the framing coming from the page, which in this case means $\operatorname{tb}\left(K_{D}\right)=0$ (see, for example, Remark 4.1 in [6], or the Legendrian realization principle of [14]). Since $\xi_{E^{\prime}}$ is a negative contact structure, we can easily isotope $K_{D}$ to another Legendrian knot so as to increase $\operatorname{tb}\left(K_{D}\right)$, so that $\operatorname{tb}\left(K_{D}\right)=1$, and hence $\operatorname{tb}\left(K_{D}\right)-1=0$, the desired framing for $D$. Thus $\left(\omega_{E^{\prime}}, J_{E^{\prime}}, \xi_{E^{\prime}}\right)$ extends over $\left(E^{\prime} \cup D, \partial\left(E^{\prime} \cup D\right)\right)$ (see Proposition [10).

In $\partial\left(E^{\prime} \cup D\right)$, the attaching circle $K_{B}$ of $B$ transversely intersects a 2-sphere at one point; make $K_{B}$ Legendrian with this property and attach $B$ along this Legendrian knot with framing $\operatorname{tb}\left(K_{B}\right)-1$, to get $\left(\omega_{E}, J_{E}, \xi_{E}\right)$ (again using Proposition 1010. (In the 1-handle $A=B^{1} \times B^{3}$, the required 2-sphere is $p \times \partial B^{3}$ for any $p \in B^{1}$.) By lemma 14, $\xi_{E}$ is overtwisted.

Since $\Sigma^{\prime}$ is symplectic, we may choose an $\omega_{E}$-compatible almost complex structure $J_{E}$ such that $\Sigma^{\prime}$ is $J_{E}$-holomorphic. Since $E^{\prime}$ is a neighborhood of $\Sigma^{\prime}, c_{1}\left(J_{E}\right) \mid E^{\prime}$ is determined by its action on $\left[\Sigma^{\prime}\right]$, which is $2-2 g^{\prime}+\alpha \cdot \alpha=$ $2-2(g+1)+\alpha \cdot \alpha$. But $i^{*}: H^{2}(E ; \mathbb{Z}) \rightarrow H^{2}\left(E^{\prime} ; \mathbb{Z}\right)$ is an isomorphism, and thus $c_{1}\left(J_{E}\right)=c \mid E$.

Now recall the more general setting in Addendum 6] We have a configuration of surfaces $\Sigma_{1}, \ldots, \Sigma_{k}$, intersecting transversely and positively, such that $\Sigma_{1}$. $\Sigma_{i}+\ldots+\Sigma_{k} \cdot \Sigma_{i}>0$. Let $E$ be a plumbed neighborhood of $\Sigma_{1} \cup \ldots \cup \Sigma_{k}$, let 
$\Sigma_{1}^{\prime}$ be the connected sum of $\Sigma_{1}$ with a trivial torus and let $E^{\prime}$ be a plumbed neighborhood of $\Sigma_{1}^{\prime} \cup \ldots \cup \Sigma_{k}$. There is a natural open book on $\partial E^{\prime}$ and two 2 -handles $B$ and $D$ which, when attached to $E^{\prime}$, give back $E$. Locally near $B$ and $D$ the picture looks exactly like Figure 8

Proposition 16 There exists a triple $\left(\omega_{E}, J_{E}, \xi_{E}\right)$ on $(E, \partial E)$ such that $\xi_{E}$ is negative and overtwisted, $\Sigma_{1}^{\prime}, \Sigma_{2}, \ldots, \Sigma_{k}$ are all $J_{E}$-holomorphic and $c_{1}\left(J_{E}\right)=$ $c \mid E$. Furthermore we can arrange that the $\omega_{E}$ area of each $\Sigma_{i}$ is $\Sigma_{1} \cdot \Sigma_{i}+\ldots+$ $\Sigma_{k} \cdot \Sigma_{i}$.

Proof Theorem 1.1 in [7] gives a construction of symplectic forms on neighborhoods of configurations exactly of the type we are dealing with here. We use this to construct $E^{\prime}$ with concave boundary; Theorem 1.1 of [7] also says that the contact structure on $\partial E^{\prime}$ is supported by the natural open book mentioned above, so that $B$ and $D$ can be attached to get an overtwisted contact boundary for $E$. Furthermore the areas of each $\Sigma_{i}$ produced in [7] are precisely the areas stated above, and the surfaces $\Sigma_{1}^{\prime}, \Sigma_{2}, \ldots, \Sigma_{k}$ are symplectic and can therefore be made $J$-holomorphic.

As mentioned in the introduction, we should now abandon all perturbations of our Morse function that have been introduced in this section, and return for the rest of this paper to the original Morse function $f$.

\section{$4 \quad$ Lutz twist cobordisms}

In this section we show how to construct a singular symplectic form on a product cobordism which connects a contact structure $\xi_{0}$ at the bottom to a contact structure $\xi_{1}$ at the top, with $\xi_{1}$ being the result of changing $\xi_{0}$ by a Lutz twist along some transverse knot.

Let $K$ be a transverse knot in some positive contact 3 -manifold. Since $K$ is transverse, it has a solid torus neighborhood which is contactomorphic to the following model. (Any two transverse knots have contactomorphic neighborhoods due to a standard Moser-Weinstein-Darboux argument.)

On the solid torus $\epsilon B^{2} \times S^{1}$, choose polar coordinates $(r, \mu), 0 \leq r \leq \epsilon$, on $\epsilon B^{2}$, and $\lambda$ on $S^{1}$. It is convenient to reparameterize by letting $\rho=r^{2} / 2$ so that $d \rho=r d r$ and $r d r d \mu d \lambda=d \rho d \mu d \lambda=d V$, the volume form for the solid torus.

Consider a $\mu \lambda$-invariant form $\alpha_{0}=f_{0}(\rho) d \mu+g_{0}(\rho) d \lambda$. Then $d \alpha_{0}=f_{0}^{\prime} d \rho d \mu+$

$g_{0}^{\prime} d \rho d \lambda$ and then $\alpha_{0} \wedge d \alpha_{0}=\left(g_{0} f_{0}^{\prime}-f_{0} g_{0}^{\prime}\right) d V>0$ implies $\frac{d}{d \rho}\left(\frac{f_{0}}{g_{0}}\right)>0$. The 
contact planes, namely $\xi_{0}=\operatorname{ker} \alpha_{0}=\operatorname{span}\left\{\frac{\partial}{\partial \rho}, g_{0}(\rho) \frac{\partial}{\partial \mu}-f_{0}(\rho) \frac{\partial}{\partial \lambda}\right\}$, should be orthogonal to the core circle $0 \times S^{1}$ parameterized by $\lambda$, so $f_{0}(0)=0$ and $g_{0}(0)=1$ is a good choice, and the map $\rho \rightarrow\left(g_{0}(\rho), f_{0}(\rho)\right)$ looks qualitatively like that drawn in Figure 9A.

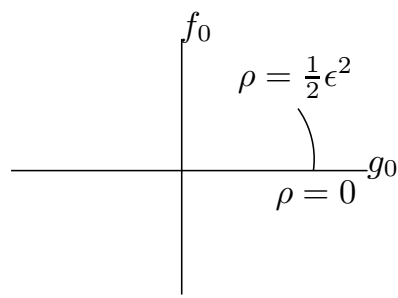

A

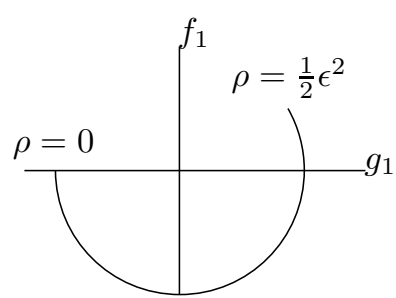

B

Figure 9: Graphs of $\rho \mapsto\left(g_{0}(\rho), f_{1}(\rho)\right)$ and $\rho \mapsto\left(g_{1}(\rho), f_{1}(\rho)\right)$

The graph of $\left(g_{0}(\rho), f_{0}(\rho)\right)$ in Figure 9A indicates that the contact planes, orthogonal to $0 \times S^{1}$, rotate in a left handed fashion as they move radially away from $0 \times S^{1}$ (left-handed because $\left\{\frac{\partial}{\partial \rho}, g_{0}(\rho) \frac{\partial}{\partial \mu}-f_{0}(\rho) \frac{\partial}{\partial \lambda}\right\}$ span the planes). We can assume they rotate only slightly as $\rho$ traverses $\left[0, \frac{1}{2} \epsilon^{2}\right]$.

Introducing a Lutz twist about $0 \times S^{1}$ means that we change the contact structure rel $\partial \epsilon B^{2} \times S^{1}$ so that the contact planes rotate left-handedly an extra $\pi$ as $\rho$ runs through $\left[0, \frac{1}{2} \epsilon^{2}\right]$, starting at $\rho=0$ orthogonal to $0 \times S^{1}$ but with the opposite orientation, and ending up in the same position as in the standard model above when $\rho=\frac{1}{2} \epsilon^{2}$ (see Figure 9B). This produces a new contact form $\alpha_{1}=f_{1}(\rho) d \mu+g_{1}(\rho) d \lambda$, which we may assume exactly equals $\alpha_{0}$ for $\rho$ near $\epsilon^{2} / 2$. Note that some authors would call this a "half Lutz twist".

On the trivial bordism $I \times \epsilon B^{2} \times S^{1}$ the standard symplectization of $\alpha_{0}$ is $d\left(e^{t} \alpha_{0}\right)=e^{t}\left(d t \wedge \alpha_{0}+d \alpha_{0}\right), t \in I$.

Proposition 17 On $I \times \epsilon B^{2} \times S^{1}$, there exists a closed 2-form $\omega$ and a metric $g$ satisfying

(1) $\omega \equiv 0$ on $Z=\frac{1}{2} \times 0 \times S^{1}$,

(2) $\omega \wedge \omega>0$ on the complement of $Z$,

(3) $\omega=d\left(e^{t} \alpha_{0}\right)$ on a neighborhood of $0 \times \epsilon B^{2} \times S^{1}$ and $I \times \partial \epsilon B^{2} \times S^{1}$,

(4) $\omega=d\left(e^{t} \alpha_{1}\right)$ on a neighborhood of $1 \times \epsilon B^{2} \times S^{1}$,

(5) $\omega$ is self-dual with respect to $g$ and transverse to the zero section of $\Lambda_{+}^{2}$. 

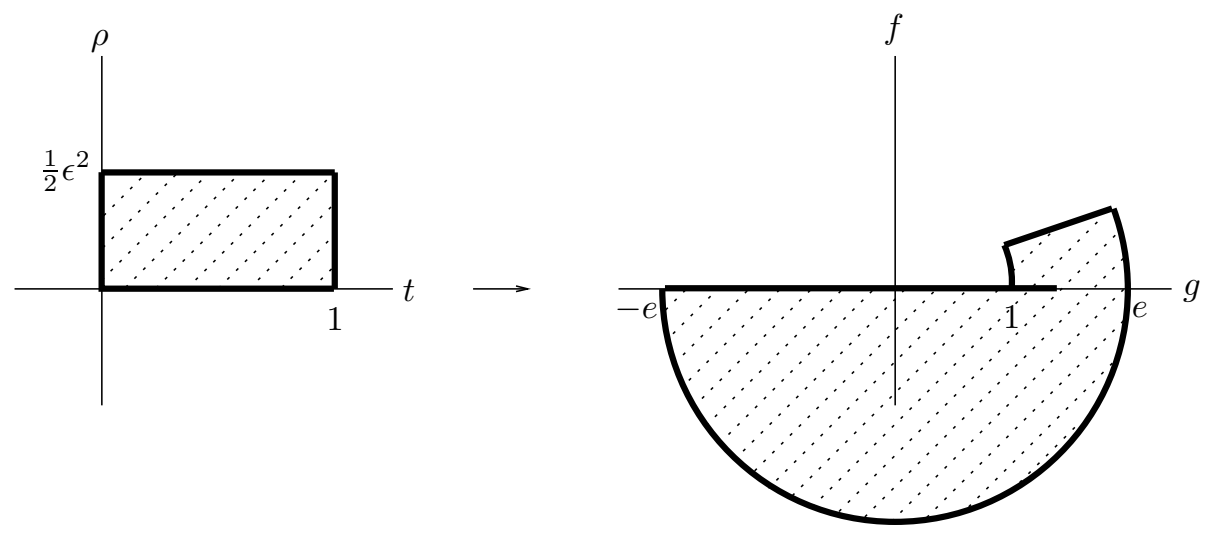

Figure 10: The map $\phi$

(6) $\omega$ and $g$ define an $\omega$-compatible almost complex structure outside a small neighborhood of $Z$.

Proof Let $\phi:[0,1] \times\left[0, \epsilon^{2} / 2\right] \hookrightarrow \mathbb{R}^{2}$ be a smooth map satisfying the following properties (see Figure 10) (we use the coordinates $(t, \rho)$ on the domain and $(g, f)$ on the range for reasons which will become clear shortly):

(1) $\phi$ is an orientation preserving immersion away from $(t=1 / 2, \rho=0)$.

(2) $\phi(t, 0)=(g(t, 0), 0)$ for all $t \in[0,1]$.

(3) On a neighborhood of $0 \times\left[0, \epsilon^{2} / 2\right]$ and $[0,1] \times \frac{1}{2} \epsilon^{2}$,

$$
\phi(t, \rho)=e^{t}\left(g_{0}(\rho), f_{0}(\rho)\right)
$$

where $g_{0}$ and $f_{0}$ are as in the preceding paragraphs.

(4) On a neighborhood of $1 \times\left[0, \epsilon^{2} / 2\right], \phi(t, \rho)=e^{t}\left(g_{1}(\rho), f_{1}(\rho)\right.$.

(5) $\phi \mid\left[0, \frac{1}{2}\right) \times 0$ moves monotically from $(1,0)$ to $(1+\delta, 0)$ for some $\delta>0$, and $\phi \mid\left(\frac{1}{2}, 1\right] \times 0$ moves monotonically from $(1+\delta, 0)$ to $(-e, 0)$.

(6) In a neighborhood of $(1 / 2,0) \mapsto(1+\delta, 0)$,

$$
\phi(t, \rho)=\left(\rho-\left(t-\frac{1}{2}\right)^{2}+1+\delta,-2 \rho\left(t-\frac{1}{2}\right)\right) .
$$

(The specified map near $(1 / 2,0) \mapsto(1+\delta, 0)$ behaves much like the complex map $z \mapsto z^{2}$, and in particular folds a half-disk neighborhood of $(1 / 2,0)$ in the upper half plane onto a disk neighborhood of $(1+\delta, 0)$.)

Letting $t$ be the coordinate on $I$, we have coordinates $\left(t, \rho=r^{2} / 2, \mu, \lambda\right)$ on $I \times \epsilon B^{2} \times S^{1}$. Writing $\phi(t, \rho)=(g(t, \rho), f(t, \rho))$, let $\alpha=f(t, \rho) d \mu+g(t, \rho) d \lambda$, 
which is a 1 -form on $I \times \epsilon B^{2} \times S^{1}$. (The fact that $f(t, 0)=0$ means that $\alpha$ is well-defined along $0 \times 0 \times S^{1}$.) Finally, let $\omega=d \alpha$. The fact that $\phi$ is an orientation preserving immersion away from $(1 / 2,0)$ implies that $\omega \wedge \omega>0$ away from $Z$. The fact that $d \phi=0$ at $(1 / 2,0)$ implies that $\omega \equiv 0$ along $Z$. The given boundary conditions for $\phi$ give the announced boundary conditions for $\omega$.

Now we must construct $g$ and verify self-duality, transversality, and compatibility with $\omega$. Near $Z$, we have

$$
\omega=d \alpha=-2 \rho d t \wedge d \mu-2 t d \rho \wedge d \mu-2 t d t \wedge d \lambda+d \rho \wedge d \lambda .
$$

Now convert polar coordinates $(r, \mu)$ (recalling that $\rho=r^{2} / 2$ ) back to cartesian coordinates $(x, y)$ on $\epsilon B^{2}$ and let $T=t-1 / 2$, to get that $\omega=y(d T \wedge d x+d y \wedge$ $d \lambda)-x(d T \wedge d y-d x \wedge d \lambda)-2 T(d x \wedge d y+d T \wedge d \lambda)$. With respect to the flat metric $g_{0}=d T^{2}+d x^{2}+d y^{2}+d \lambda^{2}$, the three sections $A=d T \wedge d x+d y \wedge d \lambda$, $B=d T \wedge d y-d x \wedge d \lambda$ and $C=d x \wedge d y+d T \wedge d \lambda$ give a frame for the bundle of self-dual 2-forms, and thus $\omega=y A-x B-2 T C$ is self-dual and transverse to the zero section.

Let $R=\sqrt{4 T^{2}+x^{2}+y^{2}}$ and let $f(R)$ be a smooth, positive function which equals $R$ for $R \geq \epsilon^{\prime}$ and equals 1 for $R \leq \epsilon^{\prime} / 2$, for some small $\epsilon^{\prime}>0$. Let $g=f(R) g_{0}$. Note that $\omega$ is still $g$-self-dual and transverse to the zero section of $\Lambda_{+}^{2}$ (since $g=g_{0}$ near $Z$ ). But now $g$ and $\omega$ induce an $\omega$-compatible almost complex structure $J$ for $R \geq \epsilon^{\prime}$, by $J=\tilde{g}^{-1} \circ \tilde{\omega}$. (Here $\tilde{g}$ and $\tilde{\omega}$ are the maps from the tangent space to the cotangent space induced by $g$ and $\omega$.) In fact, in local coordinates $(T, x, y, \lambda)$ on $R \geq \epsilon^{\prime}, \tilde{g}=R I$ (where $I$ is the identity matrix) and $\tilde{\omega}$ can be calculated from the explicit form of $\omega$ in the preceding paragraph, to get that $\tilde{\omega}$ is the matrix:

$$
Q=\left[\begin{array}{cccc}
0 & -y & x & 2 T \\
y & 0 & 2 T & -x \\
-x & -2 T & 0 & -y \\
-2 T & x & y & 0
\end{array}\right]
$$

Then $J=(1 / R) Q, J^{2}=(1 / R)^{2} Q^{2}=-I$ and $\omega(J v, J w)=v^{T} J^{T} Q J w$, with $J^{T} Q J=-(1 / R)^{2} Q^{3}=Q$, so that $\omega(J v, J w)=\omega(v, w)$.

Remark 18 In the proof above, we can rewrite $\omega$ near $Z$ as $d \lambda \wedge(2 T d T-$ $x d x-y d y)+(-2 T d x \wedge d y-x d T \wedge d y+y d T \wedge d x)=d \lambda \wedge d h+\star_{3} d h$, where $h=-\frac{1}{2} x^{2}-\frac{1}{2} y^{2}+T^{2}$ and $\star_{3}$ is the Hodge star operator on $\mathbb{R}^{3}$ with the flat metric $d y^{2}+d x^{2}+d T^{2}$. (Note that $(y, x, T)$ is the correct orientation for $\mathbb{R}^{3}$ here because we are now writing our 4 -manifold as $S^{1} \times \mathbb{R}^{3}$.) This is exactly the oriented local model given by Honda [13. 
Remark 19 If $\xi_{0}$ is a negative contact structure, we can also perform a Lutz twist along a transverse knot to get an overtwisted negative contact structure $\xi_{1}$. One might expect a similar singular symplectic cobordism from $\xi_{0}$ on the bottom to $\xi_{1}$ on the top. Upside down, this would be a cobordism from a positive contact structure to a positive contact structure which eliminates a Lutz twist. There does exist a singular almost complex structure on such a cobordism, but it is not clear how to construct a singular symplectic form with the desired properties. Much of this paper would be simplified if such a 2 -form could be constructed.

Now recall the notation from the main construction in the introduction.

Proposition 20 There exists a triple $\left(\omega_{1}, J_{1}, \xi_{1}\right)$ on $\left(X_{1}, Y_{1}\right)$, with the following properties:

(1) $\omega_{1}$ vanishes on a union of circles $Z \subset f^{-1}[0.9,1]$.

(2) $J_{1}$ is defined on $X_{1}-Z$.

(3) $\xi_{1}$ is positive and overtwisted.

(4) $Z$ consists of one unknotted circle $Z_{i}$ in each of $n$ levels between $Y_{0.9}$ and $Y_{1}$.

(5) The obstruction to extending $J_{1}$ across a 4-ball neighborhood $B_{i}$ of a disk bounded by $Z_{i}$ is $l_{i}$.

(6) The metric defined by $\omega_{1}$ and $J_{1}$ can be modified in a small neighborhood of $Z$ to be a metric on all of $X_{1}$ such that $\omega$ is $g$-self-dual and transverse to the zero section of $\Lambda_{+}^{2}$.

Proof First build $\left(\omega_{0.9}, J_{0.9}, \xi_{0.9}\right)$ on $\left(X_{0.9}, Y_{0.9}\right)$ using standard symplectic 0 - and 1-handles, as discussed in Section 2. Proposition 10. Choose numbers $0.9=a(1)<\ldots<a(n+1)=1$. Choose $K_{1} \subset\left(Y_{0.9}, \xi_{0.9}\right)$ to be a transverse unknot with $\operatorname{lk}\left(K_{1}\right)=l_{1}=-1$. First put a standard symplectification of $\xi_{0.9}$ on $f^{-1}[0.9, a(2)] \cong[0,1] \times Y_{0.9}$, and then replace this symplectification with a singular symplectic form on $[0,1] \times T_{1}$, as constructed in Proposition 17, for a small neighborhood $T_{1}$ of $K_{1}$. (This is possible because the symplectification and the singular form constructed in Proposition [17 agree on $\partial\left([0,1] \times T_{1}\right)$.) This gives $\xi_{a(2)}$ on $Y_{a(2)}$ which is overtwisted. Now we can choose $K_{2}$ a transverse unknot in $\left(Y_{a(2)}, \xi_{a(2)}\right)$ to have $\operatorname{lk}\left(K_{2}\right)=l_{2}$, and repeat. We will prove the statement about the obstruction to extending $J_{1}$ in the next section (lemma 25). Note that the metrics coming from Proposition 17 fit together smoothly because, away from $Z$, they are defined by the symplectic and almost complex structures, which fit together smoothly. 
Remark 21 There is an alternate construction for $\left(\omega_{1}, J_{1}, \xi_{1}\right)$ as follows. Begin with the disjoint union of several copies of $S^{1} \times B^{3}$, on each of which we put the singular symplectic form $d t \wedge d f+\star_{3} d f$ for $f=-x^{2}+y^{2}+z^{2}$ on $B^{3}$. The boundary is convex and overtwisted. Connect these together with 1-handles, preserving convexity on the boundary, and then kill each $S^{1}$ with a Legendrian 2 -handle to get $B^{4}$, and then attach extra 1 -handles as necessary. Then one needs to understand how the obstruction to extending $J_{1}$ across various balls depends on the Legendrian 2-handles used to kill the $S^{1}$ 's. In the end this construction should be equivalent to that outlined above.

\section{$5 \quad$ Extending almost complex structures}

Consider an almost complex structure $J$ on $T S^{3} \oplus \epsilon^{1}$ where $\epsilon^{1}$ is a trivial line bundle which we identify with the normal bundle $\nu$ to $S^{3}=\partial B^{4}$. Also let $\nu$ denote the outward unit normal vector field spanning the bundle $\nu$. We define four invariants of $J$ up to homotopy:

(1) Trivialize $T S^{3}$ using a right-invariant quaternionic frame and thus identify unit vectors in $T S^{3}$ with points in $S^{2}$. With respect to this trivialization, $J(\nu)$ then gives a map $S^{3} \rightarrow S^{2}$, and thus an element $h(J) \in$ $\pi_{3}\left(S^{2}\right)=\mathbb{Z}$.

(2) Using coordinates $(t, x, y, z)$ on $B^{4}, J\left(\frac{\partial}{\partial t}\right)$ gives a map $S^{3} \rightarrow S^{2}$, where $S^{2}$ is now the unit $(x, y, z)$ sphere. This gives an element $h^{\prime}(J) \in$ $\pi_{3}\left(S^{2}\right)=\mathbb{Z}$.

(3) Using coordinates $(t, x, y, z)$ on $B^{4}$, let $u, v, w$ be any field of frames for $\operatorname{span}\left(\frac{\partial}{\partial x}, \frac{\partial}{\partial y}, \frac{\partial}{\partial z}\right)$. Now, at each point in $S^{3}$, interpret $J\left(\frac{\partial}{\partial t}\right)$ as a point in the $(u, v, w)$ sphere, giving another element $h^{\prime \prime}(J) \in \pi_{3}\left(S^{2}\right)=\mathbb{Z}$.

(4) Choose some 4 -manifold $W$ with $\partial W=S^{3}$ such that $J$ extends over $W$. This gives the invariant $\theta(J)=\left(c_{1}(J)^{2}-2 \chi(W)-3 \sigma(W)\right) / 4$.

Remark 22 The invariant $h^{\prime}(J)$ is simply the obstruction to extending $J$ across $B^{4}$. Thus if we have an almost complex structure defined on the complement of two balls $B_{1}$ and $B_{2}$ in a 4 -manifold $X$, and $B$ is a ball containing $B_{1}$ and $B_{2}$, then $h^{\prime}(J \mid \partial B)=h^{\prime}\left(J \mid \partial B_{1}\right)+h^{\prime}\left(J \mid \partial B_{2}\right)$. Conversely, if $J$ is an almost complex structure on $\partial B$ and we choose two integers $k_{1}, k_{2}$ with $k_{1}+k_{2}=h^{\prime}(J)$, we can put two balls $B_{1}$ and $B_{2}$ in $B$ and extend $J$ across $B-\left(B_{1} \cup B_{2}\right)$ so that $h^{\prime}\left(J \mid \partial B_{i}\right)=k_{i}$. 
Lemma 23 These invariants are related by $h(J)=h^{\prime}(J)=h^{\prime \prime}(J)$ and $\theta(J)=$ $-h(J)-\frac{1}{2}$ and each uniquely characterizes $J$ up to homotopy.

Proof A direct computation shows that $h(J)=h^{\prime}(J)$. Since the field of frames $\left(\frac{\partial}{\partial x}, \frac{\partial}{\partial y}, \frac{\partial}{\partial z}\right)$ is homotopic to any other field of frames $(u, v, w)$ on $B^{4}$, we get that $h^{\prime}(J)=h^{\prime \prime}(J)$.

Note that $J$ defines an oriented plane field $\xi$ on $S^{3}$, the field of $J$-complex tangencies to $S^{3}$. The homotopy class of $J$ is uniquely determined by the homotopy class of $\xi$.

That $\theta(J)=-h(J)-\frac{1}{2}$ follows from Section 4 of $[9$ : We are really looking at invariants of $\xi ; \theta(J)$ is Gompf's $\theta(\xi)$. The invariant $h(\xi)=h(J)$ can be defined with respect to any trivialization of $T S^{3}$. Whichever trivialization we choose, there is a canonical $\mathbb{Z}$ action on homotopy classes of oriented plane fields which adds 1 to $h(\xi)$, and Gompf proves (Theorem 4.5 in [9]) that adding 1 to $h(\xi)$ corresponds to subtracting 1 from $\theta(\xi)$. Let $\xi_{0}$ be the standard tight positive contact structure on $S^{3}$. Direct calculation shows that $h\left(\xi_{0}\right)=0$ (for our particular trivialization of $\left.T S^{3}\right)$ and that $\theta\left(\xi_{0}\right)=-\frac{1}{2}$. Thus $\theta(J)=-h(J)-\frac{1}{2}$.

Finally it is well known that $h(\xi)$ is a complete invariant for homotopy classes of oriented plane fields on $S^{3}$.

Lemma 24 Given any closed $X^{4}$ with balls $B_{1}, \ldots, B_{n} \subset X$ and an almost complex structure $J$ on $X-\left(B_{1} \cup \ldots \cup B_{n}\right)$, let $d(J)=\left(c_{1}(J)^{2}-2 \chi(X)-\right.$ $3 \sigma(X)) / 4$. Then $d(J)=\sum_{i=1}^{n} h\left(J \mid \partial B_{i}\right)$.

Proof By Remark 22, we can assume that $k_{i}=h\left(J \mid \partial B_{i}\right)= \pm 1$ for each $i$. Let $X^{\prime}=X \#^{n} \mathbb{C} P^{2}$, formed by replacing each $B_{i}$ with the complement of a ball in $\mathbb{C} P^{2}$. Let $E_{i}$ be the $i$ 'th new generator in $H_{2}\left(X^{\prime} ; \mathbb{Z}\right)$ coming from the $i$ 'th $\mathbb{C} P^{2}-B^{4}$. On the $i$ 'th $\mathbb{C} P^{2}-B^{4}$, put an almost complex structure $J_{i}$ such that $c_{1}\left(J_{i}\right) \cdot E_{i}=-2 k_{i}+1$. We claim that $J$ and $J_{i}$ are homotopic on $\partial B_{i}$, so that we can glue them together to get an almost complex structure $J^{\prime}$ on all of $X^{\prime}$. This is true because $\theta\left(J_{i} \mid \partial B_{i}\right)=-k_{i}-\frac{1}{2}=-h\left(J \mid \partial B_{i}\right)-\frac{1}{2}=\theta\left(J \mid \partial B_{i}\right)$.

Now, since $J^{\prime}$ is defined on all of $X^{\prime}$, we know (by [11]) that $0=d\left(J^{\prime}\right)=$ $d(J)-\sum_{i=1}^{n} k_{i}=d(J)-\sum_{i=1}^{n} h\left(J \mid \partial B_{i}\right)$, and hence $d(J)=\sum_{i=1}^{n}\left(h \mid \partial B_{i}\right)$.

Now we want to understand the obstruction $o\left(Z_{i}\right)$ to extending $J$ across a ball containing a component $Z_{i}$ of the singular locus $Z$ for an almost complex structure $J$ coming from our Lutz twist construction. Suppose $U$ is a transverse 
unknot in a 3 -manifold with positive contact structure $\xi$, with a chosen vector field $w$ normal to $\xi$ and tangent to $U$. Let $D$ be a 2 -disk bounded by $K$ and let $B$ be a 3 -ball neighborhood of $D$. Let $\xi^{\prime}$ be the result of performing a Lutz twist along $U$, with vector field $w^{\prime}$ which is normal to $\xi^{\prime}$, tangent to $U$, and agrees with $w$ outside a neighborhood of $U$. The relevant almost complex structure $J$ is (up to homotopy) defined on $\partial(I \times B)$ as follows: On $(0 \times B) \cup(I \times \partial B), J\left(\frac{\partial}{\partial t}\right)=w$ and $J$ preserves $\xi$, and on $1 \times B, J\left(\frac{\partial}{\partial t}\right)=w^{\prime}$ and $J$ preserves $\xi^{\prime}$.

Lemma 25 With definitions as above, $o(\{0.5\} \times U)=h(J)=\operatorname{lk}(U)$.

Proof Recall that $\mathrm{lk}(U)$ is determined by choosing any nonzero section $u$ of $\xi \mid D$ and measuring the framing of $U$ given by $u \mid U$ relative to the canonical zero-framing of $U$ coming from $D$.

Extend $u$ to a section of $\xi$ on all of $B$ and let $v=J u$. We will compute $h^{\prime \prime}(J)$ using the frame $(u, v, w)$ on $B$ and the Thom-Pontrjagin construction. At each point $(t, p)$ on $\partial(I \times B)$, we write $J_{(t, p)}\left(\frac{\partial}{\partial t}\right)=a(t, p) u+$ $b(t, p) v+c(t, p) w$, normalized so that $\|(a, b, c)\|=1$, giving a map $\phi:(t, p) \mapsto$ $(a(t, p), b(t, p), c(t, p)) \in S^{2}$. Let $q=(0,0,-1)$ and $q^{\prime}=(-0.1,0,-1)$ normalized to be in $S^{2}$. We want to compute the framed cobordism class of $L=\phi^{-1}(q)$ framed by $L^{\prime}=\phi^{-1}\left(q^{\prime}\right)$. On $(0 \times B) \cup(I \times \partial B), \phi$ maps everything to $(0,0,1)$. On $1 \times B, L$ is exactly $U$, and $L^{\prime}$ is a parallel copy of $U$ realizing the framing given by $u$. Thus $h(J)=h^{\prime \prime}(J)=\operatorname{lk}(U)$.

More generally, suppose we have any harmonic 2-form $\omega$ on $X$ (with respect to some metric $g$ ) which is transverse to 0 and that $Z$ is a single unknotted component of the zero locus, with the orientable local model for $\omega$ near $Z$ (see Remark 31). Now we do not have in mind a particular contact 3-manifold $M$ near $Z$ or a Morse function with $Z$ lying in a regular level set. However we can still choose a 2 -disk $D$ bounded by $Z$ (disjoint from the rest of the zero locus) and a 4-ball neighborhood $B$ of $D$ and let $o(Z, D)$ be the obstruction to extending $J$ across $B$. Although it is not necessary for this construction, it would be nice to see how to compute $o(Z, D)$ by looking directly at the behavior of $J$ along $D$.

For this we prescribe the manner in which $D$ approaches $Z$, since $\omega$ induces a natural splitting of the normal bundle to $Z$ into 1 - and 2-dimensional subbundles. Choose local, oriented coordinates $(\theta, u, v, w)$ near $Z$, with $\theta \in S^{1}$ and $(u, v, w) \in B^{3}$, such that $\omega=d \theta \wedge d f+\star_{3} d f, f=\left(-u^{2}-v^{2}+w^{2}\right) / 2$ and $\star_{3}$ is the Hodge star operator on $B^{3}$ with metric $d u^{2}+d v^{2}+d w^{2}$ and orientation 
$(u, v, w)$. Note that our choice to make $f$ have index 2 at 0 fixes an orientation of $Z$, given by $\theta$. Let $D$ be an oriented, imbedded 2 -disk with $\partial D=Z$ with respect to this orientation, such that, near $Z, D$ coincides with the annulus $\{(\theta, u, v, w) \mid v=w=0, u \geq 0\}$. Note that $D$ has a singular foliation with singularities corresponding to complex and anticomplex points (points $p$ where $J\left(T_{p} D\right)=T_{p} D$ and $J$ agrees with or disagrees with the orientation of $\left.D\right)$. The foliation can be defined by splitting $T X \mid D$ as $\nu D \oplus T D(\nu D$ being the normal bundle to $D$ ), choosing a section $V$ of $\nu D$, homotoping $J$ to be compatible with a product metric on $\nu D \oplus T D$, and projecting $J(V)$ onto $T D$ to get a vector field on $D$ which we then integrate to get an oriented singular foliation. Thus, generically, both complex and anticomplex points can have either elliptic or hyperbolic neighborhoods. With our boundary conditions on $D$, we will see that $D$ is complex on a neighborhood of $\partial D$, so that the foliation is not generic there. However, we may make the foliation generic away from this neighborhood. With respect to this foliation, let $e_{-}$be the number of elliptic anticomplex points and let $h_{-}$be the number of hyperbolic anticomplex points.

Proposition 26 In the above situation, $o(Z, D)=-1+2\left(e_{-}-h_{-}\right)$.

Proof A model for a neighborhood of $D$ is $W=D_{1+\epsilon}^{2} \times D_{\epsilon}^{2}$ (where $D_{r}^{2}$ is the disk of radius $r$ and $S_{r}^{1}=\partial D_{r}^{1}$ ), with $D=D_{1}^{2} \times\{0\}$ and $Z=S_{1}^{1} \times\{0\}$. Let $(x, y)$ be cartesian coordinates on $D_{1+\epsilon}^{2}$ and $(z, t)$ be cartesion coordinates on $D_{\epsilon}^{2}$. Let $(r, \theta)$ be polar coordinates on $D_{1+\epsilon}^{2}$. The coordinates in the discussion above on a neighborhood of $Z$ are then $(\theta, u=1-r, v=z, w=t)$. Assume $J$ is compatible with the metric $d x^{2}+d y^{2}+d z^{2}+d t^{2}$ and let $\phi:(W-Z) \rightarrow S^{2}$ be the map given by seeing $J\left(\partial_{t}\right)$ as a point in the unit $\left(\partial_{x}, \partial_{y}, \partial_{t}\right)$-sphere.

Consider the following subsets of $W$ (see Figure 11):

(1) $A=\{(r, \theta, z, t) \mid 1-\epsilon \leq r \leq 1+\epsilon\}$

(2) $B=\{(r, \theta, z, t) \mid 0 \leq r \leq 1-\epsilon, z=t=0\}$

(3) $C=\partial A \cap \partial W$

(4) $E=\left\{(r, \theta, z, t) \mid 0 \leq r \leq 1-\epsilon, \sqrt{z^{2}+t^{2}}=\epsilon\right\}=D_{1-\epsilon}^{2} \times S_{\epsilon}^{1} \subset \partial W$

(5) $F=\{(r, \theta, z, t) \mid r=1-\epsilon\}=S_{1-\epsilon}^{1} \times D_{\epsilon}^{2} \subset \partial A$.

Note that $\partial W=C \cup E$. Let $\pi: \partial W \rightarrow B \cup C \cup F=B \cup \partial A$ be the map defined as follows: On $C, \pi$ is the identity map. On $E, \pi$ maps $\{1-2 \epsilon \leq r \leq 1-\epsilon\}$ onto $F,\{1-3 \epsilon \leq r \leq 1-2 \epsilon\}$ onto $\{1-\epsilon \leq r \leq 1-3 \epsilon, z=t=0\} \subset B$ and $\{0 \leq r \leq 1-3 \epsilon\}$ onto $\{0 \leq r \leq 1-3 \epsilon, z=t=0\} \subset B$, as indicated in Figure 12, Let $\psi=\phi \circ \pi: \partial W \rightarrow S^{2}$, and note that $\psi$ is homotopic to $\phi \mid \partial W$. 


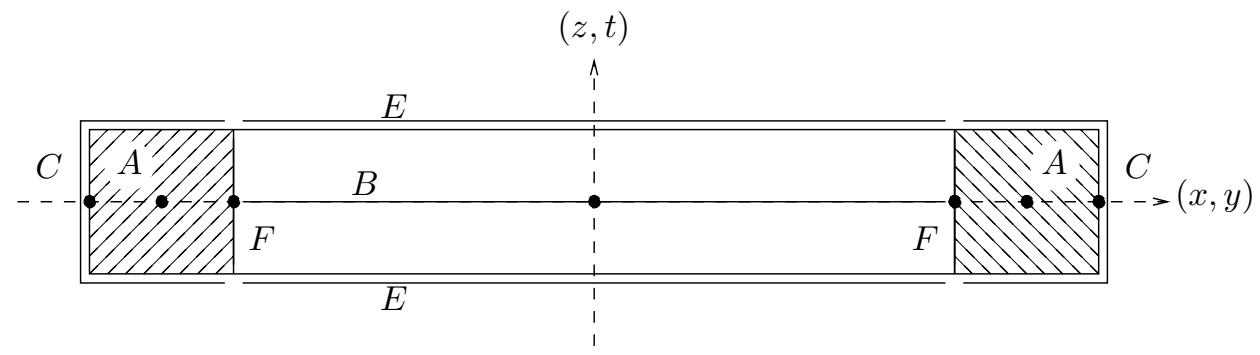

Figure 11: Various labelled subsets of $W$

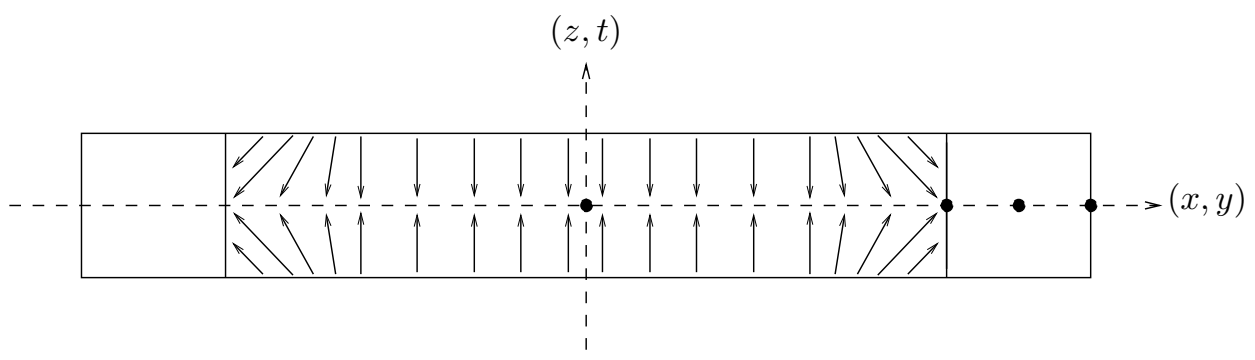

Figure 12: The projection $\pi: \partial W \rightarrow B \cup C \cup F$

We will compute $o(Z, D)$ as the oriented framed cobordism class of the oriented link $\psi^{-1}(0,0,-1)$. From the local form $\omega=d \theta \wedge d f+\star_{3} d f$ near $Z$, with $f=\left(-u^{2}-v^{2}+t^{2}\right) / 2=\left(-(1-r)^{2}-z^{2}+t^{2}\right) / 2$, we compute that, on $A, J\left(\partial_{t}\right)=$ $\left(z \partial_{r}-t \partial_{\theta}+(1-r) \partial_{z}\right) / \rho$, where $\rho=\sqrt{(1-r)^{2}+z^{2}+t^{2}}$. Thus, on $\partial A$, the set of points where $J\left(\partial_{t}\right)=-\partial_{z}$ is just $L_{0}=\{(r, \theta, z, t) \mid r=1+\epsilon, z=t=0\}=$ $S_{1+\epsilon}^{1} \times\{0\} \subset C \subset \partial W$. Furthermore, as $(\phi \mid \partial A)^{-1}(0,0,-1)=\psi^{-1}(0,0,-1) \cap C$, $L_{0}$ has framing -1 and is oriented in the negative $\theta$ direction.

On $E, \psi^{-1}(0,0,-1)$ is $\pi^{-1}\left(\phi^{-1}(0,0,-1)\right)$. Since $\phi^{-1}(0,0,-1) \cap F=\emptyset$, we are only interested in $\phi^{-1}(0,0,-1) \cap B$, which is precisely the set of anticomplex points in the original disk $D$. Each such anticomplex point $\left(x_{i}, y_{i}\right)$ becomes a circle $L_{i}=\left\{\left(x_{i}, y_{i}, z, t\right) \mid \sqrt{z^{2}+t^{2}}=\epsilon\right\}$ in $E$ with framing 0 , oriented against the orientation of $S_{\epsilon}^{1}=\partial D_{\epsilon}^{2}$ if $\left(x_{i}, y_{i}\right)$ is elliptic, and with the orientation of $S_{\epsilon}^{1}$ if $\left(x_{i}, y_{i}\right)$ is hyperbolic. Thus the complete oriented framed link $\psi^{-1}(0,0,-1)$ is a -1 -framed unknot $L_{0}$ with $n 0$-framed meridians $L_{1}, \ldots, L_{n}$ (where $D$ has $n$ anticomplex points), with $\operatorname{lk}\left(L_{0}, L_{i}\right)=+1$ (respctively -1$)$ if $\left(x_{i}, y_{i}\right)$ is elliptic (respectively hyperbolic); see Figure 13] This is framed cobordant to an unknot with framing equal to the sum of the entries in the linking matrix, which is $-1+2 e_{-}-2 h_{-}$. 


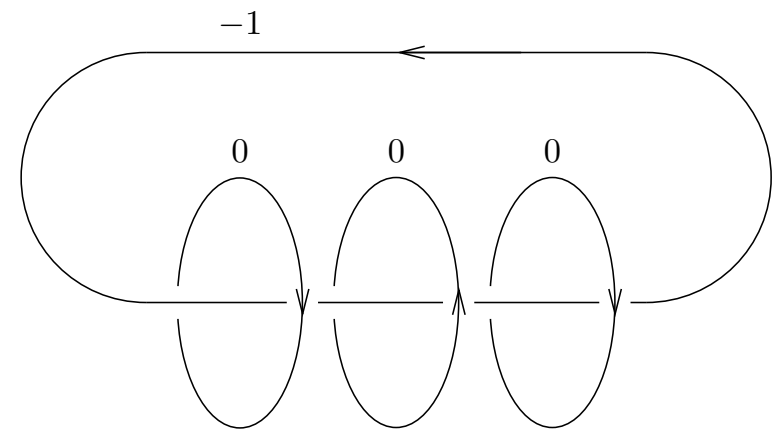

Figure 13: $\psi^{-1}(0,0,-1)$, in the case of two elliptic anticomplex points and one hyperbolic anticomplex point

\section{Cocycles that guide our construction}

Recall that $X_{1}$ is the union of the 0 - and 1-handles of $X$, and that we have a triple $\left(\omega_{1}, J_{1}, \xi_{1}\right)$ on $\left(X_{1}, Y_{1}\right)$, with zero circles $Z=Z_{1} \cup \ldots \cup Z_{n} \subset \operatorname{int}\left(X_{1}\right)$. As in the introduction, fix a trivialization $\tau$ of $\xi_{1}$; this can be done because $c_{1}\left(\xi_{1}\right)=c_{1}\left(J_{1} \mid Y_{1}\right)=c_{1}\left(J_{1}\right) \mid Y_{1}=0$, since $H^{2}\left(X_{1} ; \mathbb{Z}\right)=0$.

Now suppose that $K_{1}, \ldots, K_{q}$, for some $q$, are disjoint Legendrian knots in $\left(Y_{1}, \xi_{1}\right)$, with rotation numbers $\operatorname{rot}\left(K_{i}\right)$ measured relative to $\tau$. Attach symplectic 2-handles $H_{1}^{2}, \ldots, H_{q}^{2}$ along $K_{1}, \ldots, K_{q}$ to produce a 4 -manifold $W$ with boundary and a triple $\left(\omega_{W}, J_{W}, \xi_{W}\right)$ on $(W, \partial W)$ that extends $\left(\omega_{1}, J_{1}, \xi_{1}\right)$. $W$ deformation retracts onto a 2 -complex, with 2 -cells $C_{1}^{2}, \ldots, C_{q}^{2}$ corresponding to the 2 -handles $H_{1}^{2}, \ldots, H_{q}^{2}$, with 1 -cells coming from the 1 -handles of $X_{1}$, and with one 0 -cell at the center of the 0 -handle. We may assume that $Z$ misses the 2 -skeleton. Thus we get a 2 -cochain $r$ on the 2 -skeleton given by $r\left(C_{i}^{2}\right)=\operatorname{rot}\left(K_{i}\right)$. This is trivially a cocycle on the 2 -skeleton (there are no 3 -cells), and therefore defines a cohomology class $[r] \in H^{2}(W ; \mathbb{Z})$. The following result is a slight generalization of a standard fact (see [9]) relating rotation numbers and Chern classes.

Lemma $27[r]=c_{1}\left(J_{W}\right)$

Proof In fact we will show that $r$ is a cocycle given by trivializing $J_{W}$ (by which we mean the $\mathbb{C}^{2}$-bundle defined by $J_{W}$ ) over the 1 -skeleton and measuring the obstruction to extending this trivialization over each 2 -cell. $\tau$ together with the outward normal $\nu$ to $\partial X_{1}$ defines a trivialization $(\tau, \nu)$ of $J_{W}$ over 
$\partial X_{1}$. By an isotopy of $W$ we can arrange that the 1 -skeleton lies in $\partial X_{1}$ and that the 2-skeleton misses the interior of $W_{1}$. Thus we take $(\tau, \nu)$ as the starting trivialization of $J_{W}$ over the 1 -skeleton. Clearly $(\tau, \nu)$ extends across as much of each 2-cell $C_{i}^{2}$ as lies in $\partial W_{1}$; the rest of $C_{i}^{2}$ is simply the core of $H_{i}^{2}$ and our task is to show that the obstruction to extending $(\tau, \nu)$ across $H_{i}^{2}$ is exactly $\operatorname{rot}\left(K_{i}\right)$. Let $\kappa$ be the unit tangent vector to $K_{i}$; in the proof of Proposition 2.3 in [9] it is shown that $(\kappa, \nu)$ extends across $H_{i}$. From this we see that the winding of $\kappa$ with respect to $\tau$ is precisely the obstruction in question.

In our main construction outlined in the introduction, we use this lemma three times, once to recognize $c_{1}\left(J_{2}^{\prime}\right)$ in our "false start" construction of $\left(\omega_{2}^{\prime}, J_{2}^{\prime}, \xi_{2}^{\prime}\right)$, and then to construct the correct $\left(\omega_{N}, J_{N}, \xi_{N}\right)$ and its extension to $\left(\omega_{2}, J_{2}, \xi_{2}\right)$. Recall that the cocycle $x$ used to determine the rotation numbers which produce $\left(\omega_{2}, J_{2}, \xi_{2}\right)$ is an honest cocycle for the full 4 -complex decomposition of $X$.

Lemma 28 Because $x$ is a cocycle, $J_{2}$ extends over the 3 -skeleton $X$.

Proof Consider a 3 -cell $C_{j}^{3}$. Since $x$ is a cocycle, $x\left(\partial C_{j}^{3}\right)=0$, which, by the same argument as in the preceding lemma, means that $c_{1}\left(J_{2} \mid \partial C_{j}^{3}\right)=$ $0 \in H^{2}\left(\partial C_{j}^{3} ; \mathbb{Z}\right)$. Trivialize $T X \mid C_{j}^{3}$ as $\mathbb{R}^{4} \times C_{j}^{3}$, with standard basis vectors $e_{1}, e_{2}, e_{3}, e_{4}$ for $\mathbb{R}^{4}$; almost complex structures on $C_{j}^{3}$ and on $\partial C_{j}^{3}$ are then determined up to homotopy by the map $J\left(e_{4}\right): \partial C_{j}^{3} \rightarrow S^{2}$, where $S^{2}$ is the unit $\left(e_{1}, e_{2}, e_{3}\right)$-sphere. For any almost complex structure $J$ on $\partial C_{j}^{3}$, it is not hard to see that $c_{1}(J) \in H^{2}\left(\partial C_{j}^{3} ; \mathbb{Z}\right)=\mathbb{Z}$ is twice the degree of $J\left(e_{4}\right)$. (This is most easily seen by noting that the $C^{2}$ bundle over $\partial C_{j}^{3}$ is the Whitney sum of a trivial complex line bundle spanned by $e_{4}$ and a complex line bundle $\xi$; but $\xi$ is visibly the tangent bundle of $S^{2}$ when $J\left(e_{4}\right)$ is the identity map, so $c_{1}(J)$ is twice the degree in the generating case.) Thus in our case $J_{2}\left(e_{4}\right)$ has degree 0 and therefore extends over $C_{j}^{3}$.

\section{$7 \quad$ Plane fields and spin $^{\mathbb{C}}$ structures}

Recall that in our main construction we had constructed two almost complex structure $J_{E}$ and $J_{*} \mid E$ on $E$, with $c_{1}\left(J_{E}\right)=c_{1}\left(J_{*} \mid E\right)$.

Lemma 29 This implies that $J_{E}$ is homotopic to $J_{*} \mid E$. 
Proof Pick a nowhere zero section $\tau$ of $T E$ (an orientable $\mathbb{R}^{4}$-bundle over a 2-complex always has a nonzero section) and let $\mathcal{J}$ be the unit $S^{2}$ bundle orthogonal to $\tau$; up to homotopy we can view $J_{E}$ and $J_{*}$ as sections of $\mathcal{J}$. (This is simply the bundle of local almost complex structures.) We can homotope $J_{E}$ to agree with $J_{*}$ over the 1 -skeleton. Over each 2 -cell $C_{i}^{2}$, we can trivialize $\mathcal{J}$ as $S^{2} \times C_{i}^{2}$ so that $J_{E}$ is a constant section. Then $J_{*}$ becomes a map from $C_{i}^{2}$ to $S^{2}$ which is constant on $\partial C_{i}^{2}$, which we can think of as a map from $S^{2}$ to $S^{2}$, and read off the degree of this map. This gives a cocycle and, much as in the preceding section, it is not hard to see that twice this cocycle represents $c_{1}\left(J_{E}\right)-c_{1}\left(J_{*}\right)$. Thus, because $H^{2}(E ; \mathbb{Z})$ has no 2 -torsion and $c_{1}\left(J_{E}\right)-c_{1}\left(J_{*}\right)=0$, we see that this cocycle is a coboundary, which implies that we can change our choice of trivialization over the 1-skeleton to make the cocycle 0 . Thus $J_{E}$ is homotopic to $J_{*} \mid E$.

Next we show how to hit the right $\operatorname{spin}^{\mathbb{C}}$ structure in our construction. Here we recall a special property of our cell decomposition of $X$, that $\partial C_{p+1}^{2}=C_{1}^{1}$, where $C_{p+1}^{2}$ is the 2 -cell associated to the 2 -handle $H_{p+1}^{2}$ coming from $E$, and $C_{1}^{1}$ is a 1 -cell. Let $b$ be the 1 -cochain that is 1 on $C_{1}^{1}$ and 0 on everything else. Then every class $a \in H^{2}(X ; \mathbb{Z})$ can be represented by a cocycle $z$ with $z\left(C_{p+1}^{2}\right)=0$; if $z\left(C_{p+1}^{2}\right) \neq 0$, then replace $z$ with $z-\left(z\left(C_{p+1}^{2}\right)\right) \delta b$.

For our purposes we will think of a $\operatorname{spin}^{\mathbb{C}}$ structure on $X$ as a homotopy class of almost complex structures over the 2 -skeleton which extends over the 3 skeleton. As mentioned above, we should think of almost complex structures as sections of an $S^{2}$-bundle $\mathcal{J}$. For our fixed class $c \in H^{2}(X ; \mathbb{Z})$, let $\mathcal{S}_{c}$ be the set of all $\operatorname{spin}^{\mathbb{C}}$ structures $s$ with $c_{1}(s)=c$. It is well known that $H^{2}(X ; \mathbb{Z})$ acts freely and transitively on the set of all $\operatorname{spin}^{\mathbb{C}}$ structures on $X$, changing $c_{1}$ by twice the cohomology class that is acting. Thus the difference between two spin $^{\mathbb{C}}$ structures $s_{0}, s_{1} \in \mathcal{S}_{c}$ is a cohomology class of order 2 .

Proposition 30 Suppose that, in our construction, we used a cocycle representative $x_{0}$ of $c$, and that this produced $s_{0} \in \mathcal{S}_{c}$. Let $a \in H^{2}(X ; \mathbb{Z})$ be the class of order 2 which acts on $s_{0}$ to give $s_{1}=s$. Choose a representative $z$ for a with $z\left(C_{p+1}^{2}\right)=0$. Then, if we repeat our construction with the cocycle $x_{1}=x_{0}-2 z$ instead of $x_{0}$, we will produce $s_{1}$ instead of $s_{0}$.

Proof Let $J_{0}, J_{1}$ be the almost complex structures produced by $x_{1}, x_{0}$, respectively. Recall that our construction depended on a fixed trivialization over the 1 -skeleton, and that we can homotope $J_{0}$ to $J_{1}$ over the 1 -skeleton. Again, over each $2-$ cell $C_{i}^{2}$ we can trivialize $\mathcal{J}$ so that $J_{0}$ is a constant section. Then 
the action of $a$ is given precisely by changing this section to a section of degree $z\left(C_{i}^{2}\right)$. The difference between the obstructions to extending the trivialization of $J_{1}=J_{0}$ on the 1 -skeleton to a trivialization of $J_{1}$ or $J_{0}$ over $C_{i}^{2}$ is twice this degree, and thus we see that changing the rotation numbers by $2 z$ implements this action of $a$.

It is important that $z\left(C_{p+1}^{2}\right)=0$ because in our final construction we discard $J_{2}$ and only use $J_{N}$. Thus, if we had changed our construction on $H_{p+1}^{2}$, the change would not survive to the end of the construction.

\section{References}

[1] C Bohr, Embedded surfaces and almost complex structures, Proc. Amer. Math. Soc. 128 (2000) 2147-2154

[2] F Ding, H Geiges, A Legendrian surgery presentation of contact 3-manifolds, arXiv:math.SG/0107045

[3] F Ding, H Geiges, A Stipsicz, Surgery diagrams for contact 3-manifolds, arXiv:math.SG/0307237

[4] Y Eliashberg, Classification of overtwisted contact structures on 3-manifolds, Invent. Math. 98 (1989) 623-637

[5] Y Eliashberg, Topological characterization of Stein manifolds of dimension $>2$, Internat. J. Math. 1 (1990) 29-46

[6] D T Gay, Explicit concave fillings of contact three-manifolds, Math. Proc. Cambridge Philos. Soc. 133 (2002) 431-441

[7] D T Gay, Open books and configurations of symplectic surfaces, Algebr. Geom. Topol. 3 (2003) 569-586

[8] N Goodman, Contact structures and open books, PhD Thesis, U. Texas Austin (2003)

[9] R E Gompf, Handlebody construction of Stein surfaces, Annals of Math. 148 (1998) 619-693

[10] RE Gompf, Spin $\mathbb{C}^{\mathbb{C}}$-structures and homotopy equivalences, Geom. Topol. 1 (1997) 41-50

[11] K Hirzebruch, H Hopf, Felder von Flächenelementen in 4-dimensionalen Mannigfaltigkeiten, Math. Ann. 136 (1958) 156-172

[12] K Honda, An openness theorem for harmonic 2-forms on 4-manifolds, Illinois J. Math. 44 (2000) 479-495

[13] K Honda, Local properties of self-dual harmonic 2-forms on a 4-manifold, arXiv:dg-ga/9705010 
[14] K Honda, On the classification of tight contact structures I, Geom. Topol. 4 (2000) 309-368

[15] G Mikhalkin, $J$-holomorphic curves in almost complex surfaces do not always minimize the genus, Proc. Amer. Math. Soc. 125 (1997) 1831-1833

[16] R Scott, Closed Self-Dual Two-Forms on Four-Dimensional Handlebodies, PhD Thesis, Harvard (2003)

[17] C H Taubes, The structure of pseudo-holomorphic subvarieties for a degenerate almost complex structure and symplectic form on $S^{1} \times B^{3}$, Geom. Topol. 2 (1998) 221-332

[18] A Weinstein, Contact surgery and symplectic handlebodies, Hokkaido Math. J. 20 (1991) no. 2, 241-251 